\title{
Complex Dynamics on the Routes to Chaos in a Discrete Predator-Prey System with Crowley-Martin Type Functional Response
}

\author{
Huayong Zhang $\mathbb{D}^{\mathbb{D}}$, Shengnan Ma, Tousheng Huang, Xuebing Cong, \\ Zichun Gao, and Feifan Zhang \\ Research Center for Engineering Ecology and Nonlinear Science, North China Electric Power University, Beijing, China \\ Correspondence should be addressed to Huayong Zhang; rceens@ncepu.edu.cn
}

Received 29 October 2017; Revised 26 January 2018; Accepted 31 January 2018; Published 29 April 2018

Academic Editor: Hassan A. El-Morshedy

Copyright (c) 2018 Huayong Zhang et al. This is an open access article distributed under the Creative Commons Attribution License, which permits unrestricted use, distribution, and reproduction in any medium, provided the original work is properly cited.

We present in this paper an investigation on a discrete predator-prey system with Crowley-Martin type functional response to know its complex dynamics on the routes to chaos which are induced by bifurcations. Via application of the center manifold theorem and bifurcation theorems, occurrence conditions for flip bifurcation and Neimark-Sacker bifurcation are determined, respectively. Numerical simulations are performed, on the one hand, verifying the theoretical results and, on the other hand, revealing new interesting dynamical behaviors of the discrete predator-prey system, including period-doubling cascades, period-2, period-3, period-4, period-5, period-6, period-7, period-8, period-9, period-11, period-13, period-15, period-16, period-20, period22, period-24, period-30, and period-34 orbits, invariant cycles, chaotic attractors, sub-flip bifurcation, sub-(inverse) NeimarkSacker bifurcation, chaotic interior crisis, chaotic band, sudden disappearance of chaotic dynamics and abrupt emergence of chaos, and intermittent periodic behaviors. Moreover, three-dimensional bifurcation diagrams are utilized to study the transition between flip bifurcation and Neimark-Sacker bifurcation, and a critical case between the two bifurcations is found. This critical bifurcation case is a combination of flip bifurcation and Neimark-Sacker bifurcation, showing the nonlinear characteristics of both bifurcations.

\section{Introduction}

Predator-prey interaction shows widespread existence in nature and can take many forms, such as resource-consumer, plant-herbivore, and phytoplankton-zooplankton forms [1]. Due to the ubiquity and importance of the predator-prey interaction between populations, the dynamics of predatorprey systems have attracted the attention of many scholars, and the research on predator-prey systems has become one of the dominant themes in ecology [2-4]. In recent decades, mathematical models have been established to analyze various complex dynamics of the predator-prey systems in various circumstances [4-6].

The Lotka-Volterra predator-prey model, proposed in the pioneering works of Lotka and Volterra [7, 8], is probably the simplest and most basic predator-prey model in the field. The development of subsequent predator-prey models is mostly based on the Lotka-Volterra model $[3,9,10]$. The development may be a change of functional response (e.g., Holling type I, II, and III classifications) or numerical response to describe different ecological processes in the predator-prey interaction $[11,12]$. Several researchers argued that the nonautonomous model is closer to the realistic predator-prey system than the autonomous model [3]. Therefore, predator-prey models with a time delay were also studied because the predator-prey interaction may have time lag [13]. In order to incorporate the influence of seasonal variation into the predator-prey interaction, predator-prey models with periodically varying parameters were also proposed and investigated [14-16].

In earlier approaches, most predator-prey models were time-continuous. However, the continuous models hardly described the dynamics when the populations have nonoverlapping generations or the number of populations is small $[17,18]$. For these cases, applying discrete-time models should be more reasonable and appropriate [17]. In recent years, 
more and more scholars have payed attention to discretetime predator-prey models. Compared with the continuous models, the discrete dynamic models often have advantages in describing richer nonlinear characteristics and complexity, such as various periodic orbits, quasiperiodic behaviors, and chaotic attractors [19-21]. Moreover, a few research works found that the discrete model can lead to more accurate results than the corresponding continuous model in describing predator-prey dynamics $[6,22,23]$.

Through the research of He and Lai [2], Liu and Xiao [19], Jing and Yang [20], Agiza et al. [5], Hu et al. [6], Huang and Zhang [21], and others, they found out that flip bifurcation and Neimark-Sacker bifurcation are two basic bifurcations occurring in discrete predator-prey systems. The two bifurcations often start the routes to chaos, revealing the dynamic transition from the nonchaotic state to the chaotic state. Moreover, complex dynamics on the routes to chaos can be observed, including cascades of period doubling, periodic windows, intermittent chaos, and chaotic crisis [19-21]. For example, via calculating Lyapunov exponents and fractal dimension, Agiza et al. determined diverse strange attractors of a predator-prey system [5].

When we study the nonlinear characteristics of predatorprey systems, the functional response of the predator to the prey is an essential factor which affects the dynamical properties. From an ecological point of view, functional responses may be determined by the prey escape capability, prey habitat property, and predator hunting capability [24]. Skalski and Gilliam [12] suggested that three classical preypredator-dependent functional responses-BeddingtonDeAngelis, Crowley-Martin, and Hassell-Varley functional responses-can provide a better description of predator feeding over a range of predator-prey abundance presence.

Particularly, Crowley-Martin functional response is much more suitable for the case where the predator feeding rate is decreased by a higher predator density even when the prey density is high [12]. The Crowley-Martin functional response depends on both the predator and the prey and takes into account the interaction between predators, regardless of whether a predator is looking for prey. It is similar to the classical Beddington-DeAngelis functional response; in particular, it shows the interspecies interference with each other. Usually, it is employed to demonstrate the predator-prey stability. And, therefore, it shows ecological significance for researching the predator-prey models with Crowley-Martin functional response. A classical predatorprey system with Crowley-Martin functional response can be described by the following equations [25]:

$$
\begin{aligned}
& \frac{d u}{d t}=u\left(a-u-\frac{b v}{(1+\alpha u)(1+\beta v)}\right)=f(u, v), \\
& \frac{d v}{d t}=v\left(-c+\frac{d u}{(1+\alpha u)(1+\beta v)}\right)=g(u, v),
\end{aligned}
$$

where $u(t)$ and $v(t)$ represent the densities of the prey and the predator, respectively; parameters $a, b, c, d, \alpha$, and $\beta$ are positive constants; the parameter $a$ is the intrinsic growth rate of the prey and the parameter $c$ is the mortality rate of the predator; $b$ and $d / b$ stand for the effect of capture rate and conversion factor denoting the newly born predators for each captured prey; the parameters $\alpha$ and $\beta$ are the saturating parameters of Crowley-Martin functional response, $\alpha$ measures the magnitude of interference among prey, and $\beta$ expresses the interference among the predators.

In recent years, the research on the predator-prey systems with Crowley-Martin functional response has attracted a lot of attention. For example, Yang studied the stability and instability of positive equilibrium in a predator-prey model with Crowley-Martin functional response and time delay [24]. Asymptotic properties of a stochastic predatorprey model with Crowley-Martin functional response were studied by Liu et al., such as global existence and stochastic boundedness of the positive solution [26]. A stagestructured predator-prey system with Crowley-Martin functional response was investigated, and the persistence of the system and global asymptotic stability of the positive equilibrium were assessed [27]. Sivakumar et. al. analyzed a diffusive density-dependent predator-prey model with Crowley-Martin functional response and revealed NeimarkSacker bifurcation, Turing bifurcation, and spatiotemporal patterns [28]. Dong et al. investigated the positive solutions of a spatiotemporal predator-prey system with Crowley-Martin functional response and obtained a complete understanding of the uniqueness and nonuniqueness of positive solutions $[29,30]$. Li and Wu discussed the extinction and persistence results of time-dependent positive solutions to the system [31]. From previous studies, it can be seen that the predatorprey model with Crowley-Martin functional response often exhibits rich dynamics and attracts the attention of many researchers. However, the complex dynamics of the discretetime predator-prey model with Crowley-Martin functional response are still in lack of research.

The discretized form of system (1a) and (1b) was never investigated in previous studies. Since the growth, death, feeding, and migration of the predator and prey individuals always occur periodically, we can observe the dynamics of the predator-prey system by a particular time scale. This time scale can be defined by the generation span of the predator and prey populations and it measures the regeneration time of both populations. Its value is mainly determined by the population types; on the other hand, it is influenced by the change of population size or environmental conditions. In this research, we denote the time scale on which predatorprey dynamics are described and observed as parameter $\tau$. Applying the forward Euler scheme with time interval $\tau$, system (1a) and (1b) is now transformed to a discrete predator-prey system:

$$
\begin{aligned}
& u(k+1)=f(u(k), v(k)) \\
& =u(k) \\
& \quad+\tau u(k)\left(a-u(k)-\frac{b v(k)}{(1+\alpha u(k))(1+\beta v(k))}\right),
\end{aligned}
$$




$$
\begin{aligned}
& v(k+1)=g(u(k), v(k)) \\
& =v(k)+\tau v(k)\left(-c+\frac{d u(k)}{(1+\alpha u(k))(1+\beta v(k))}\right),
\end{aligned}
$$

where $k$ represents the sequence number of iterations. If we give the initial time as $t_{0}$, the $k$ th iteration represents the time at $t_{0}+k \tau$.

In recent decades, the research on discrete predator-prey systems has become an important topic. Via the research of Li and $\mathrm{Wu}$ [31], the dynamical properties of system (1a) and (1b) have been revealed a lot. However, nonlinear characteristics of discrete system (2a) and (2b) are still scarcely known. In the literature, Ren et al. have actually investigated a Leslie-Gower type discrete predator-prey model with Crowley-Martin functional response and found the existence of Marotto chaos [32]. They also controlled chaotic orbits to be a fixed point by a feedback control method. Different from the study of Ren et al. [32], this research focuses on a detailed exploration of the complex dynamics, such as periodic windows, subbifurcations, and chaos crisis, on the routes to chaos induced by the bifurcations of system (2a) and (2b).

In order to facilitate the study of system (2a) and (2b), we rewrite the equations of system (2a) and (2b) as a map; that is,

$$
\left(\begin{array}{l}
u \\
v
\end{array}\right) \rightarrow\left(\begin{array}{c}
u+\tau u\left(a-u-\frac{b v}{(1+\alpha u)(1+\beta v)}\right) \\
v+\tau v\left(-c+\frac{d u}{(1+\alpha u)(1+\beta v)}\right)
\end{array}\right) .
$$

In this research, the bifurcations and complex dynamics of the discrete predator-prey system with Crowley-Martin functional response are explored through map (3). The exploration is arranged as follows. Section 2 will give the stability analysis on the fixed points of map (3). Section 3 will provide bifurcation analysis and determine occurrence conditions for flip bifurcation and Neimark-Sacker bifurcation. Section 4 will present numerical simulations for complex dynamic behaviors of the discrete predator-prey system. Section 5 will describe the conclusions.

\section{Stability Analysis}

The fixed points of map (3) can be calculated through the following equations:

$$
\begin{aligned}
& u=u+\tau u\left(a-u-\frac{b v}{(1+\alpha u)(1+\beta v)}\right), \\
& v=v+\tau v\left(-c+\frac{d u}{(1+\alpha u)(1+\beta v)}\right),
\end{aligned}
$$

which directly yield

$$
\begin{gathered}
\left(u_{0}, v_{0}\right):(0,0) \\
\left(u_{1}, v_{1}\right):(a, 0) ; \\
\left(u_{2}, v_{2}\right):\left(u^{*}, v^{*}\right),
\end{gathered}
$$

where $u^{*}$ is a positive root of the following cubic equations:

$$
\begin{aligned}
& \alpha \beta d u^{3}+\beta d(1-\alpha a) u^{2}+(b d-\beta a d-\alpha c b) u-c b \\
& =0 \text {, } \\
& v^{*}=\frac{d\left(a-u^{*}\right) u^{*}}{c b} .
\end{aligned}
$$

According to previous results in the literature [21], the sufficient conditions for map (3) possessing a unique positive fixed point are determined as follows:

$$
\begin{gathered}
(1-\alpha a)\left(2 \beta d(1-\alpha a)^{2}-18 \alpha \beta d(1-\alpha a)\right. \\
-9 \alpha(b d-\beta a d-\alpha c d))-27 \alpha^{2} c d<0 .
\end{gathered}
$$

To determine the stability of the fixed points of map (3), the Jacobian matrix associated with the map is applied and can be described as follows:

$$
J(u, v)=\left[\begin{array}{cc}
1+\tau\left(a-2 u-\frac{b v}{(1+\alpha u)^{2}(1+\beta v)}\right) & -\tau \frac{b u}{(1+\alpha u)(1+\beta v)^{2}} \\
\tau \frac{d v}{(1+\alpha u)^{2}(1+\beta v)} & 1+\tau\left(-c+\frac{d u}{(1+\alpha u)(1+\beta v)^{2}}\right)
\end{array}\right]
$$

For the Jacobian matrix of (8), we substitute the values of the three fixed points into it and calculate the two eigenvalues, $\lambda_{1}$ and $\lambda_{2}$. If $\left|\lambda_{1}\right|<1$ and $\left|\lambda_{2}\right|<1$, the corresponding fixed point is considered to be stable; if $\left|\lambda_{1}\right|>1$ or $\left|\lambda_{2}\right|>1$, an unstable fixed point emerges. For the fixed point $\left(u_{0}, v_{0}\right)$, the corresponding Jacobian matrix is

$$
J\left(u_{0}, v_{0}\right)=\left[\begin{array}{cc}
1+a \tau & 0 \\
0 & 1-c \tau
\end{array}\right],
$$

the two eigenvalues of which are $1+a \tau$ and $1-c \tau$. Since $1+a \tau$ is larger than 1 , then $\left(u_{0}, v_{0}\right)$ is unstable. For the fixed point $\left(u_{1}, v_{1}\right)$, the Jacobian matrix is obtained as

$$
J\left(u_{1}, v_{1}\right)=\left[\begin{array}{cc}
1-a \tau & -\tau \frac{a b}{1+\alpha a} \\
0 & 1+\tau\left(-c+\frac{d a}{1+\alpha a}\right)
\end{array}\right] .
$$


The two eigenvalues of $J\left(u_{1}, v_{1}\right)$ are $1-a \tau$ and $1-c \tau+d a \tau /(1+$ $\alpha a)$. When the following conditions are established,

$c+\alpha a c-a d>0$,

$$
0<\tau<\min \left\{\frac{2}{a}, \frac{2(c(1+\alpha a)-d a)}{1+\alpha a}\right\},
$$

the two eigenvalues are both less than one. Therefore, under conditions (11), $\left(u_{1}, v_{1}\right)$ is stable. The Jacobian matrix of $\left(u_{2}, v_{2}\right)$ is presented as the following matrix:

$$
\begin{aligned}
J\left(u_{2}, v_{2}\right) & =\left[\begin{array}{ll}
a_{11} & a_{12} \\
a_{21} & a_{22}
\end{array}\right] \\
& =\left[\begin{array}{cc}
1+\tau\left(a-2 u^{*}\right)-\frac{\tau b d\left(a-u^{*}\right) u^{*}}{\left(1+\alpha u^{*}\right)^{2}\left(c b+\beta d u^{*}\left(a-u^{*}\right)\right)} & -\frac{\tau c^{2} b^{3} u^{*}}{\left(1+\alpha u^{*}\right)\left(c b+\beta d u^{*}\left(a-u^{*}\right)\right)^{2}} \\
\frac{\tau d^{2} u^{*}\left(a-u^{*}\right)}{\left(1+\alpha u^{*}\right)^{2}\left(c b+\beta d u^{*}\left(a-u^{*}\right)\right)} & 1-\tau c+\frac{\tau b^{2} c^{2} d u^{*}}{\left(1+\alpha u^{*}\right)\left(c b+\beta d u^{*}\left(a-u^{*}\right)\right)^{2}}
\end{array}\right] .
\end{aligned}
$$

Likewise, the two eigenvalues of matrix (3) are

$$
\lambda_{1,2}=\frac{1}{2}\left(-p \pm \sqrt{p^{2}-4 q}\right)
$$

where

$$
\begin{aligned}
p= & -2-\tau b^{*}, \\
q= & 1+\tau b^{*}+\tau^{2} a^{*}, \\
a^{*} & =\left(a-2 u^{*}-\frac{b d\left(a-u^{*}\right) u^{*}}{\left(1+\alpha u^{*}\right)^{2}\left(c b+\beta d\left(a-u^{*}\right) u^{*}\right)}\right) \\
& \cdot\left(-c+\frac{b^{2} c^{2} d u^{*}}{\left(1+\alpha u^{*}\right)\left(c b+\beta d\left(a-u^{*}\right) u^{*}\right)^{2}}\right) \\
& +\frac{b^{3} c^{2} d^{2}\left(a-u^{*}\right) u^{* 2}}{\left(1+\alpha u^{*}\right)^{3}\left(c b+\beta d\left(a-u^{*}\right) u^{*}\right)^{3}}, \\
b^{*} & =a-2 u^{*}-c-\frac{b d\left(a-u^{*}\right) u^{*}}{\left(1+\alpha u^{*}\right)^{2}\left(c b+\beta d\left(a-u^{*}\right) u^{*}\right)} \\
& +\frac{b^{2} c^{2} d u^{*}}{\left(1+\alpha u^{*}\right)\left(c b+\beta d\left(a-u^{*}\right) u^{*}\right)^{2}} .
\end{aligned}
$$

We calculate the stability conditions of $\left(u_{2}, v_{2}\right)$, that is, $\left|\lambda_{1}\right|<$ 1 and $\left|\lambda_{2}\right|<1$, as follows:

$$
\begin{aligned}
q & <1, \\
-(1+q) & <p<1+q .
\end{aligned}
$$

\section{Bifurcation Analysis}

3.1. Flip Bifurcation. Around the stable fixed point $\left(u_{2}, v_{2}\right)$, flip bifurcation of map (3) is studied via the flip bifurcation theorem and center manifold theorem [27]. According to the flip bifurcation theorem, the predator-prey system undergoes flip bifurcation when one of the two eigenvalues of $\left(u_{2}, v_{2}\right)$ is equal to -1 . Therefore, it is explicitly said that $q=p-1$; that is,

$$
1+\tau b^{*}+\tau^{2} a^{*}=-2-\tau b^{*}-1
$$

which directly yields

$$
\tau^{*}=\frac{4}{-b^{*} \mp \sqrt{b^{* 2}-4 a^{*}}} .
$$

At such critical point $\tau^{*}$, the two eigenvalues of $\left(u_{2}, v_{2}\right)$ are $\lambda_{1}=1$ and $\lambda_{2}=1-p$. Moreover, the value of $\lambda_{2}$ should satisfy $\left|\lambda_{2}\right| \neq 1$; that is,

$$
\begin{gathered}
\tau^{*}\left(c+2 u^{*}-a+\frac{b d\left(a-u^{*}\right) u^{*}}{\left(1+\alpha u^{*}\right)^{2}\left(c b+\beta d\left(a-u^{*}\right) u^{*}\right)}\right. \\
\left.-\frac{b^{2} c^{2} d u^{*}}{\left(1+\alpha u^{*}\right)\left(c b+\beta d\left(a-u^{*}\right) u^{*}\right)^{2}}\right) \neq 2,4 .
\end{gathered}
$$

Consider the bifurcation parameter $\tau$ also as a dependent variable. We then translate the fixed point $\left(u_{2}, v_{2}, \tau^{*}\right)$ to the origin via the following translation:

$$
\begin{aligned}
& w=u-u^{*}, \\
& z=v-v^{*}, \\
& \tilde{\tau}=\tau-\tau^{*} .
\end{aligned}
$$

Consequently, via Taylor expansion near the fixed point, map (3) can be transformed as 


$$
\begin{aligned}
& \left(\begin{array}{l}
w \\
z \\
\tilde{\tau}
\end{array}\right) \\
& \longrightarrow\left(\begin{array}{r}
a_{11} w+a_{12} z+\frac{1}{2} a_{13} w^{2}+\frac{1}{2} a_{14} z^{2}+a_{15} w z+a_{16} w \tilde{\tau}+a_{17} z \tilde{\tau}+\frac{1}{6} a_{18} w^{3}+\frac{1}{2} a_{19} w z^{2}+\frac{1}{2} a_{110} w^{2} z+\frac{1}{6} a_{11} z^{3}+\frac{1}{2} a_{112} w^{2} \tilde{\tau}+\frac{1}{2} a_{113} z^{2} \tilde{\tau}+a_{114} w z \tilde{\tau}+o\left((|w|+|z|+|\tilde{\tau}|)^{4}\right) \\
a_{21} w+a_{22} z+\frac{1}{2} a_{23} w^{2}+\frac{1}{2} a_{24} z^{2}+a_{25} w z+a_{26} w \tilde{\tau}+a_{27} z \tilde{\tau}+\frac{1}{6} a_{28} w^{3}+\frac{1}{2} a_{29} w z^{2}+\frac{1}{2} a_{210} w^{2} z+\frac{1}{6} a_{211} z^{3}+\frac{1}{2} a_{212} w^{2} \tilde{\tau}+\frac{1}{2} a_{213} z^{2} \tilde{\tau}+a_{214} w z \tilde{\tau}+o\left((|w|+|z|+|\tilde{\tau}|)^{4}\right) \\
\tilde{\tau}
\end{array}\right),
\end{aligned}
$$

where $o\left((|w|+|z|+|\tilde{\tau}|)^{4}\right)$ is a polynomial with at least four orders with variables $|w|,|z|$, and $|\widetilde{\tau}|$. The coefficients $a_{i j}$ can be calculated by

$$
\begin{aligned}
& a_{1 j}=\left.\frac{\partial^{s_{1}} f}{\partial u^{s_{1}}} \frac{\partial^{s_{2}} f}{\partial v^{s_{2}}} \frac{\partial^{s_{3}} f}{\partial \tau^{s_{3}}}\right|_{(u, v, \tau)=\left(u^{*}, v^{*}, \tau^{*}\right)}, \\
& a_{2 j}=\left.\frac{\partial^{s_{1}} g}{\partial u^{s_{1}}} \frac{\partial^{s_{2}} g}{\partial v^{s_{2}}} \frac{\partial^{s_{3}} g}{\partial \tau^{s_{3}}}\right|_{(u, v, \tau)=\left(u^{*}, v^{*}, \tau^{*}\right)},
\end{aligned}
$$

where $s_{1}, s_{2}$, and $s_{3}$ are the orders of the variables $w, z$, and $\tilde{\tau}$, respectively, in the corresponding term of $a_{i j}$. Here, we define $\partial^{0} g / \partial u^{0}=1$.

Applying a reversible transformation as

$$
\left(\begin{array}{l}
w \\
z
\end{array}\right) \longrightarrow\left(\begin{array}{cc}
a_{12} & a_{12} \\
-1-a_{11} & \lambda_{2}-a_{11}
\end{array}\right)\left(\begin{array}{c}
\widetilde{w} \\
\widetilde{z}
\end{array}\right)
$$

map (20) becomes

$$
\begin{aligned}
\left(\begin{array}{c}
\tilde{w} \\
\tilde{z} \\
\tilde{\tau}
\end{array}\right) \longrightarrow & \left(\begin{array}{ccc}
-1 & 0 & 0 \\
0 & \lambda_{2} & 0 \\
0 & 0 & 1
\end{array}\right)\left(\begin{array}{c}
\widetilde{w} \\
\tilde{z} \\
\tilde{\tau}
\end{array}\right) \\
& +\frac{1}{a_{12}\left(1+\lambda_{2}\right)}\left(\begin{array}{c}
F_{1}(\widetilde{w}, \widetilde{z}, \tilde{\tau}) \\
F_{2}(\widetilde{w}, \widetilde{z}, \tilde{\tau}) \\
0
\end{array}\right),
\end{aligned}
$$

where

$$
\begin{aligned}
F_{1} & (\widetilde{w}, \widetilde{z}, \widetilde{\tau})=a_{12}^{2}\left(\frac{a_{13}\left(\lambda_{2}-a_{11}\right)}{2}-\frac{a_{12} a_{23}}{2}\right)(\widetilde{w}+\widetilde{z})^{2} \\
& +\left(\frac{a_{14}\left(\lambda_{2}-a_{11}\right)}{2}-\frac{a_{12} a_{24}}{2}\right) \\
& \cdot\left(\left(\lambda_{2}-a_{11}\right) \tilde{z}-\left(1+a_{11}\right) \widetilde{w}\right)^{2} \\
& +a_{12}\left(a_{15}\left(\lambda_{2}-a_{11}\right)-a_{12} a_{25}\right)(\widetilde{w}+\widetilde{z}) \\
& \cdot\left(\left(\lambda_{2}-a_{11}\right) \widetilde{z}-\left(1+a_{11}\right) \widetilde{w}\right) \\
& +a_{12}\left(a_{16}\left(\lambda_{2}-a_{11}\right)-a_{12} a_{26}\right)(\widetilde{w}+\widetilde{z}) \widetilde{\tau} \\
& +\left(a_{17}\left(\lambda_{2}-a_{11}\right)-a_{12} a_{27}\right) \\
& \cdot\left(\left(\lambda_{2}-a_{11}\right) \widetilde{z}-\left(1+a_{11}\right) \widetilde{w}\right) \tilde{\tau}
\end{aligned}
$$

$$
\begin{aligned}
& +a_{12}^{3}\left(\frac{a_{18}\left(\lambda_{2}-a_{11}\right)}{6}-\frac{a_{12} a_{28}}{6}\right)(\widetilde{w}+\widetilde{z})^{3} \\
& +a_{12}\left(\frac{a_{19}\left(\lambda_{2}-a_{11}\right)}{2}-\frac{a_{12} a_{29}}{2}\right)(\widetilde{w}+\widetilde{z}) \\
& \cdot\left(\left(\lambda_{2}-a_{11}\right) \widetilde{z}-\left(1+a_{11}\right) \widetilde{w}\right)^{2} \\
& +a_{12}^{2}\left(\frac{a_{110}\left(\lambda_{2}-a_{11}\right)}{2}-\frac{a_{12} a_{210}}{2}\right)(\widetilde{w}+\widetilde{z})^{2} \\
& \cdot\left(\left(\lambda_{2}-a_{11}\right) \widetilde{z}-\left(1+a_{11}\right) \widetilde{w}\right) \\
& +\left(\frac{a_{111}\left(\lambda_{2}-a_{11}\right)}{6}-\frac{a_{12} a_{211}}{6}\right) \\
& \cdot\left(\left(\lambda_{2}-a_{11}\right) \widetilde{z}-\left(1+a_{11}\right) \widetilde{w}\right)^{3} \\
& +a_{12}^{2}\left(\frac{a_{112}\left(\lambda_{2}-a_{11}\right)}{2}-\frac{a_{12} a_{212}}{2}\right)(\widetilde{w}+\widetilde{z})^{2} \tilde{\tau} \\
& +\left(\frac{a_{113}\left(\lambda_{2}-a_{11}\right)}{2}-\frac{a_{12} a_{213}}{2}\right) \\
& \cdot\left(\left(\lambda_{2}-a_{11}\right) \widetilde{z}-\left(1+a_{11}\right) \widetilde{w}\right)^{2} \widetilde{\tau} \\
& +a_{12}\left(a_{114}\left(\lambda_{2}-a_{11}\right)-a_{12} a_{214}\right)(\widetilde{w}+\widetilde{z}) \\
& \cdot\left(\left(\lambda_{2}-a_{11}\right) \widetilde{z}-\left(1+a_{11}\right) \widetilde{w}\right) \widetilde{\tau} \\
& +o\left((|\widetilde{w}|+|\widetilde{z}|+|\widetilde{\tau}|)^{4}\right),
\end{aligned}
$$

$$
\begin{aligned}
& F_{2}(\widetilde{w}, \widetilde{z}, \widetilde{\tau})=a_{12}^{2}\left(\frac{a_{13}\left(1+a_{11}\right)}{2}+\frac{a_{12} a_{23}}{2}\right)(\widetilde{w}+\widetilde{z})^{2} \\
& +\left(\frac{a_{14}\left(1+a_{11}\right)}{2}+\frac{a_{12} a_{24}}{2}\right) \\
& +\left(\left(\lambda_{2}-a_{11}\right) \widetilde{z}-\left(1+a_{11}\right) \widetilde{w}\right)^{2} \\
& +a_{12}\left(a_{15}\left(1+a_{11}\right)+a_{12} a_{25}\right)(\widetilde{w}+\widetilde{z}) \\
& +\left(\left(\lambda_{2}-a_{11}\right) \widetilde{z}-\left(1+a_{11}\right) \widetilde{w}\right) \\
& +a_{12}\left(a_{16}\left(; 21+a_{11}\right)+a_{12} a_{26}\right)(\widetilde{w}+\widetilde{z}) \widetilde{\tau} \\
& +\left(a_{17}\left(1+a_{11}\right)+a_{12} a_{27}\right)
\end{aligned}
$$




$$
\begin{aligned}
& \cdot\left(\left(\lambda_{2}-a_{11}\right) \widetilde{z}-\left(1+a_{11}\right) \widetilde{w}\right) \widetilde{\tau} \\
& +a_{12}^{3}\left(\frac{a_{18}\left(1+a_{11}\right)}{6}+\frac{a_{12} a_{28}}{6}\right)(\widetilde{w}+\widetilde{z})^{3} \\
& +a_{12}\left(\frac{a_{19}\left(1+a_{11}\right)}{2}+\frac{a_{12} a_{29}}{2}\right)(\widetilde{w}+\widetilde{z}) \\
& \cdot\left(\left(\lambda_{2}-a_{11}\right) \widetilde{z}-\left(1+a_{11}\right) \widetilde{w}\right)^{2} \\
& +a_{12}^{2}\left(\frac{a_{110}\left(1+a_{11}\right)}{2}+\frac{a_{12} a_{210}}{2}\right)(\widetilde{w}+\widetilde{z})^{2} \\
& \cdot\left(\left(\lambda_{2}-a_{11}\right) \tilde{z}-\left(1+a_{11}\right) \widetilde{w}\right) \\
& +\left(\frac{a_{111}\left(1+a_{11}\right)}{6}+\frac{a_{12} a_{211}}{6}\right) \\
& \cdot\left(\left(\lambda_{2}-a_{11}\right) \widetilde{z}-\left(1+a_{11}\right) \widetilde{w}\right)^{3} \\
& +a_{12}^{2}\left(\frac{a_{112}\left(1+a_{11}\right)}{2}+\frac{a_{12} a_{212}}{2}\right)(\widetilde{w}+\widetilde{z})^{2} \tilde{\tau} \\
& +\left(\frac{a_{113}\left(1+a_{11}\right)}{2}+\frac{a_{12} a_{213}}{2}\right) \\
& \cdot\left(\left(\lambda_{2}-a_{11}\right) \widetilde{z}-\left(1+a_{11}\right) \widetilde{w}\right)^{2} \widetilde{\tau} \\
& +a_{12}\left(a_{114}\left(1+a_{11}\right)+a_{12} a_{214}\right)(\widetilde{w}+\widetilde{z}) \\
& \cdot\left(\left(\lambda_{2}-a_{11}\right) \widetilde{z}-\left(1+a_{11}\right) \widetilde{w}\right) \widetilde{\tau} \\
& +o\left((|\widetilde{w}|+|\widetilde{z}|+|\widetilde{\tau}|)^{4}\right) \text {. }
\end{aligned}
$$

The center manifold of map (23) at the fixed point $(0,0,0)$ is then determined. According to the center manifold theorem, a central manifold at the fixed point $(0,0,0)$ can be approximated by the following:

$$
\begin{aligned}
& W^{C}(0,0,0)=\left\{(\widetilde{w}, \widetilde{z}, \widetilde{\tau}) \in R^{3} \mid \widetilde{z}=h^{*}(\widetilde{w}, \widetilde{\tau}), h^{*}(0,0)\right. \\
& \left.\quad=0, D h^{*}(0,0)=0\right\}
\end{aligned}
$$

where $h^{*}(\widetilde{w}, \widetilde{\tau})$ is assumed to be

$$
\begin{aligned}
h^{*}(\widetilde{w}, \widetilde{\tau})= & e_{0} \widetilde{\tau}+e_{1} \widetilde{w}^{2}+e_{2} \widetilde{w} \widetilde{\tau}+e_{3} \widetilde{\tau}^{2} \\
& +o\left((|\widetilde{w}|+|\widetilde{\tau}|)^{3}\right) .
\end{aligned}
$$

Utilizing map (23) on both sides of $\widetilde{z}=h^{*}(\widetilde{w}, \widetilde{\tau})$ leads to

$$
\begin{aligned}
e_{0} \widetilde{\tau}+ & e_{1}\left(-\widetilde{w}+\frac{F_{1}\left(\widetilde{w}, h^{*}(\widetilde{w}, \widetilde{\tau}), \widetilde{\tau}\right)}{a_{12}\left(1+\lambda_{2}\right)}\right)^{2} \\
& +e_{2} \tilde{\tau}\left(-\widetilde{w}+\frac{F_{1}\left(\widetilde{w}, h^{*}(\widetilde{w}, \widetilde{\tau}), \widetilde{\tau}\right)}{a_{12}\left(1+\lambda_{2}\right)}\right)+e_{3} \widetilde{\tau}^{2}
\end{aligned}
$$

$$
\begin{aligned}
& -\lambda_{2}\left(e_{0} \tilde{\tau}+e_{1} \widetilde{w}^{2}+e_{2} \widetilde{w} \widetilde{\tau}+e_{3} \tilde{\tau}^{2}\right) \\
& -\frac{F_{2}\left(\widetilde{w}, h^{*}(\widetilde{w}, \widetilde{\tau}), \widetilde{\tau}\right)}{a_{12}\left(1+\lambda_{2}\right)}=o\left((|\widetilde{w}|+|\widetilde{\tau}|)^{3}\right),
\end{aligned}
$$

simultaneously balancing the variables in (28); then, we get $\mathrm{v}$ $e_{0}=e_{3}=0$, and the expressions of $e_{1}$ and $e_{2}$ are

$$
\begin{aligned}
e_{1} & =\frac{1}{2 a_{12}\left(1-\lambda_{2}^{2}\right)}\left(a_{12}^{2}\left(a_{13}\left(1+a_{11}\right)+a_{12} a_{23}\right)\right. \\
& +\left(a_{14}\left(1+a_{11}\right)+a_{12} a_{24}\right)\left(1+a_{11}\right)^{2} \\
& \left.-2 a_{12}\left(a_{15}\left(1+a_{11}\right)+a_{12} a_{25}\right)\left(1+a_{11}\right)\right), \\
e_{2} & =\frac{1}{a_{12}\left(1+\lambda_{2}\right)^{2}}\left(a_{17}\left(1+a_{11}\right)^{2}\right. \\
& \left.+a_{12}\left(a_{27}-a_{16}\right)\left(1+a_{11}\right)-a_{12}^{2} a_{26}\right) .
\end{aligned}
$$

Considering the dynamics of map (23) restricted in the center manifold $W^{C}(0,0,0)$, hence we obtain a onedimensional mapping as

$$
\begin{aligned}
F: \widetilde{w} \longrightarrow & -\widetilde{w}+\mu_{1} \widetilde{w}^{2}+\mu_{2} \widetilde{w} \widetilde{\tau}+\mu_{3} \widetilde{w}^{2} \widetilde{\tau}+\mu_{4} \widetilde{w} \widetilde{\tau}^{2} \\
& +\mu_{5} \widetilde{w}^{3}+o\left((|\widetilde{w}|+|\widetilde{\tau}|)^{4}\right) .
\end{aligned}
$$

The coefficients in map (30) are described as follows:

$$
\begin{aligned}
\mu_{1} & =\frac{1}{a_{12}\left(1+\lambda_{2}\right)}\left(\frac{a_{12}^{2}}{2}\left(a_{13}\left(\lambda_{2}-a_{11}\right)-a_{12} a_{23}\right)\right. \\
& +\frac{1}{2}\left(a_{14}\left(\lambda_{2}-a_{11}\right)-a_{12} a_{24}\right)\left(1+a_{11}\right)^{2} \\
& \left.-a_{12}\left(a_{15}\left(\lambda_{2}-a_{11}\right)-a_{12} a_{25}\right)\left(1+a_{11}\right)\right), \\
\mu_{2} & =\frac{1}{1+\lambda_{2}}\left(a_{16}\left(\lambda_{2}-a_{11}\right)-a_{12} a_{26}\right) \\
& -\frac{\left(1+a_{11}\right)}{a_{12}\left(1+\lambda_{2}\right)}\left(a_{17}\left(\lambda_{2}-a_{11}\right)-a_{12} a_{27}\right), \\
\mu_{3} & =\frac{1}{a_{12}\left(1+\lambda_{2}\right)}\left[a_{12}^{2}\left(a_{13}\left(\lambda_{2}-a_{11}\right)-a_{12} a_{23}\right) e_{2}\right. \\
& -\left(a_{14}\left(\lambda_{2}-a_{11}\right)-a_{12} a_{24}\right)\left(\lambda_{2}-a_{11}\right)\left(1+a_{11}\right) e_{2} \\
& +a_{12}\left(a_{15}\left(\lambda_{2}-a_{11}\right)-a_{12} a_{25}\right)\left(\lambda_{2}-2 a_{11}-1\right) e_{2} \\
& +a_{12}\left(a_{16}\left(\lambda_{2}-a_{11}\right)-a_{12} a_{26}\right) e_{1} \\
& +\left(a_{17}\left(\lambda_{2}-a_{11}\right)-a_{12} a_{27}\right)\left(\lambda_{2}-a_{11}\right) e_{1} \\
& +a_{12}^{2}\left(\frac{a_{112}\left(\lambda_{2}-a_{11}\right)}{2}-\frac{a_{12} a_{212}}{2}\right)
\end{aligned}
$$




$$
\begin{aligned}
& +\left(\frac{a_{113}\left(\lambda_{2}-a_{11}\right)}{2}-\frac{a_{12} a_{213}}{2}\right)\left(1+a_{11}\right)^{2} \\
& \left.-a_{12}\left(a_{114}\left(\lambda_{2}-a_{11}\right)-a_{12} a_{214}\right)\left(1+a_{11}\right)\right], \\
\mu_{4} & =\frac{e_{2}}{1+\lambda_{2}}\left(a_{16}\left(\lambda_{2}-a_{11}\right)-a_{12} a_{26}\right) \\
& +\frac{e_{2}\left(\lambda_{2}-a_{11}\right)}{a_{12}\left(1+\lambda_{2}\right)}\left(a_{17}\left(\lambda_{2}-a_{11}\right)-a_{12} a_{27}\right), \\
\mu_{5} & =\frac{1}{a_{12}\left(1+\lambda_{2}\right)}\left[a_{12}^{2}\left(a_{13}\left(\lambda_{2}-a_{11}\right)-a_{12} a_{23}\right) e_{1}\right. \\
& -\left(a_{14}\left(\lambda_{2}-a_{11}\right)-a_{12} a_{24}\right)\left(\lambda_{2}-a_{11}\right)\left(1+a_{11}\right) e_{1} \\
& +a_{12}\left(a_{15}\left(\lambda_{2}-a_{11}\right)-a_{12} a_{25}\right)\left(\lambda_{2}-2 a_{11}-1\right) e_{1} \\
& \left.+\left(\frac{a_{111}\left(\lambda_{2}-a_{11}\right)}{6}-\frac{a_{12} a_{211}}{6}\right)\left(1+a_{11}\right)^{3}\right] \\
& +a_{12}^{3}\left(\frac{a_{18}\left(\lambda_{2}-a_{11}\right)}{6}-\frac{a_{12} a_{28}}{6}\right) \\
& +a_{12}\left(\frac{a_{19}\left(\lambda_{2}-a_{11}\right)}{2}-\frac{a_{12} a_{29}}{2}\right)\left(1+a_{11}\right)^{2} \\
& \left.+a_{11}\right) \\
& \\
& \left.=\frac{a_{12} a_{210}}{2}\right)\left(1+a_{11}\right) \\
&
\end{aligned}
$$

On the basis of map (30), the predator-prey system experiencing flip bifurcation needs the requirement of the following two conditions:

$$
\eta_{1}=\left(\frac{\partial^{2} F}{\partial \widetilde{w} \partial \tilde{\tau}}+\frac{1}{2} \frac{\partial F}{\partial \widetilde{\tau}} \frac{\partial^{2} F}{\partial \widetilde{w}^{2}}\right) \neq 0
$$

$$
\begin{aligned}
& \left(\begin{array}{l}
w \\
z
\end{array}\right) \\
& \longrightarrow\left(\begin{array}{l}
a_{11} w+a_{12} z+\frac{1}{2} a_{13} w^{2}+\frac{1}{2} a_{14} z^{2}+a_{15} w z+\frac{1}{6} a_{18} w^{3}+\frac{1}{2} a_{19} w z^{2}+\frac{1}{2} a_{110} w^{2} z+\frac{1}{6} a_{111} z^{3}+o\left((|w|+|z|)^{4}\right) \\
a_{21} w+a_{22} z+\frac{1}{2} a_{23} w^{2}+\frac{1}{2} a_{24} z^{2}+a_{25} w z+\frac{1}{6} a_{28} w^{3}+\frac{1}{2} a_{29} w z^{2}+\frac{1}{2} a_{210} w^{2} z+\frac{1}{6} a_{211} z^{3}+o\left((|w|+|z|)^{4}\right)
\end{array}\right) .
\end{aligned}
$$

$$
\begin{aligned}
\eta_{2}=\left(\frac{1}{6} \frac{\partial^{3} F}{\partial \widetilde{w}^{3}}+\left(\frac{1}{2} \frac{\partial^{2} F}{\partial \widetilde{w}^{2}}\right)^{2}\right) \neq 0, & \\
& (\widetilde{w}=0, \tilde{\tau}=0),
\end{aligned}
$$

which are equivalent to

$$
\begin{aligned}
& \eta_{1}=\mu_{2} \neq 0, \\
& \eta_{2}=\mu_{5}+\mu_{1}^{2} \neq 0 .
\end{aligned}
$$

Hence, the occurrence of flip bifurcation at the fixed point $\left(u_{2}, v_{2}\right)$ with parameter $\tau$ varying in a small neighborhood of $\tau^{*}$ needs the satisfaction of conditions (18) and (33). Furthermore, if $\eta_{2}>0$, a period-2 orbit bifurcates from $\left(u_{2}, v_{2}\right)$ and is stable; if $\eta_{2}<0$, the period- 2 orbit bifurcating from $\left(u_{2}, v_{2}\right)$ is unstable.

3.2. Neimark-Sacker Bifurcation. When map (3) undergoes Neimark-Sacker bifurcation, the two eigenvalues of the fixed point $\left(u_{2}, v_{2}\right)$ are a pair of conjugate complex numbers whose module is one. Hence, we directly have $p^{2}-4 q<0$ and $q=1$, which are further determined as

$$
\begin{aligned}
b^{* 2}-4 a^{*} & <0, \\
\tau_{0} & =-\frac{b^{*}}{a^{*}} .
\end{aligned}
$$

Under conditions (34), we translate the fixed point $\left(u_{2}, v_{2}\right)$ to the origin by the following translation:

$$
\begin{aligned}
& w=u-u^{*}, \\
& z=v-v^{*} .
\end{aligned}
$$

With application of Taylor expansion, map (3) is transformed to
The coefficients in the map are also calculated by (21) but $(u, v, \tau)=\left(u^{*}, v^{*}, \tau^{*}\right)$. The two eigenvalues at the fixed point $(0,0)$ of map $(36)$ are also a pair of conjugate complexes with a module of one. These two eigenvalues are written as

$$
\begin{aligned}
\lambda\left(\tau_{0}\right), \bar{\lambda}\left(\tau_{0}\right) & =\frac{-p\left(\tau_{0}\right)}{2} \pm \frac{\tilde{i}}{2} \sqrt{4 q\left(\tau_{0}\right)-p^{2}\left(\tau_{0}\right)} \\
& =\theta \pm \tilde{i} \varphi
\end{aligned}
$$

where $\tilde{i}=\sqrt{-1}$ and $|\lambda|=\sqrt{q\left(\tau_{0}\right)}=1$. The modulus of the two eigenvalues satisfies

$$
\begin{aligned}
\gamma & =\left.\frac{\mathrm{d}|\lambda(\tau)|}{\mathrm{d} \tau}\right|_{\tau=\tau_{0}} \\
& =\left.\frac{1}{2} \frac{1}{\sqrt{a^{*} \tau^{2}+b^{*} \tau+1}}\left(2 a^{*} \tau+b^{*}\right)\right|_{\tau=\tau_{0}=-b^{*} / a^{*}} \\
& =-\frac{b^{*}}{2} \neq 0 .
\end{aligned}
$$


Simultaneously, the two eigenvalues should not be real numbers or imaginary numbers, which means $\left(\lambda\left(\tau_{0}\right)\right)^{n} \neq 1$, $n=1,2,3,4$. Since $q\left(\tau_{0}\right)=1$, we have $p\left(\tau_{0}\right) \neq-2,0,1,2$. In the meantime, $p^{2}\left(\tau_{0}\right)<4 q\left(\tau_{0}\right)=4$. Therefore, $p\left(\tau_{0}\right) \neq 0,1$, which is equivalent to

$$
\begin{gathered}
\tau_{0}\left(c+2 u^{*}-a+\frac{b d\left(a-u^{*}\right) u^{*}}{\left(1+\alpha u^{*}\right)^{2}\left(c b+\beta d\left(a-u^{*}\right) u^{*}\right)}\right. \\
\left.-\frac{b^{2} c^{2} d u^{*}}{\left(1+\alpha u^{*}\right)\left(c b+\beta d\left(a-u^{*}\right) u^{*}\right)^{2}}\right) \neq 2,3 .
\end{gathered}
$$

Through the transformation

$$
\left(\begin{array}{l}
w \\
z
\end{array}\right) \rightarrow\left(\begin{array}{cc}
a_{11} & 0 \\
\theta-a_{11} & -\varphi
\end{array}\right)\left(\begin{array}{l}
\widetilde{w} \\
\widetilde{z}
\end{array}\right)
$$

the normal form of map (36) is obtained as

$$
\left(\begin{array}{c}
\widetilde{w} \\
\widetilde{z}
\end{array}\right) \longrightarrow\left(\begin{array}{cc}
\theta & -\varphi \\
\varphi & \theta
\end{array}\right)\left(\begin{array}{l}
\widetilde{w} \\
\widetilde{z}
\end{array}\right)+\frac{1}{a_{12} \varphi}\left(\begin{array}{l}
G_{1}(\widetilde{w}, \widetilde{z}) \\
G_{2}(\widetilde{w}, \widetilde{z})
\end{array}\right),
$$

in which

$$
\begin{aligned}
& G_{1}(\widetilde{w}, \widetilde{z})=\left(\frac{a_{12}^{2} a_{13}}{2} \varphi+\frac{a_{14}}{2} \varphi\left(\theta-a_{11}\right)^{2}\right. \\
& \left.+a_{12} a_{15}\left(\theta-a_{11}\right) \varphi\right) \widetilde{w}^{2}+\frac{a_{14}}{2} \varphi^{3} z^{2} \\
& \quad-\left(a_{14}\left(\theta-a_{11}\right) \varphi^{2}+a_{12} a_{15} \varphi^{2}\right) \widetilde{w} z+\left(\frac{a_{12}^{3} a_{18}}{6}\right. \\
& +\frac{a_{12} a_{19}}{2} \varphi\left(\theta-a_{11}\right)^{2}+\frac{a_{12}^{2} a_{110}}{2} \varphi\left(\theta-a_{11}\right) \\
& \left.+\frac{1}{6} a_{111} \varphi\left(\theta-a_{11}\right)^{3}\right) \widetilde{w}^{3}+\left(\frac{a_{12} a_{19}}{2} \varphi^{3}\right. \\
& \left.+\frac{a_{111}}{2} \varphi^{3}\left(\theta-a_{11}\right)\right) \widetilde{w} \widetilde{z}^{2}-\left(a_{12} a_{19}\left(\theta-a_{11}\right) \varphi^{2}\right. \\
& \left.+\frac{a_{12}^{2} a_{110}}{2} \varphi^{2}+\frac{a_{111}}{2}\left(\theta-a_{11}\right)^{2} \varphi^{2}\right) \widetilde{w}^{2} \widetilde{z}-\frac{a_{111}}{6} \\
& +\varphi^{4} \widetilde{z}^{3}+o\left((|\widetilde{w}|+|\widetilde{z}|)^{4}\right), \\
& G_{2}(\widetilde{w}, \widetilde{z})=\left(\frac{a_{12}^{2}}{2}\left(a_{13}\left(\theta-a_{11}\right)-a_{12} a_{23}\right)\right.
\end{aligned}
$$

$+\frac{1}{2}\left(a_{14}\left(\theta-a_{11}\right)-a_{12} a_{24}\right)\left(\theta-a_{11}\right)^{2}$

$\left.+a_{12}\left(\theta-a_{11}\right)\left(a_{15}\left(\theta-a_{11}\right)-a_{12} a_{25}\right)\right) \widetilde{w}^{2}$

$+\left(\frac{a_{14}\left(\theta-a_{11}\right)}{2}-\frac{a_{12} a_{24}}{2}\right) \varphi^{2} \widetilde{z}^{2}$

$-\left(\left(a_{14}\left(\theta-a_{11}\right)-a_{12} a_{24}\right)\left(\theta-a_{11}\right) \varphi\right.$

$\left.+a_{12}\left(a_{15}\left(\theta-a_{11}\right)-a_{12} a_{25}\right) \varphi\right) \widetilde{w z}$

$+\left(\frac{a_{12}^{3}}{6}\left(a_{18}\left(\theta-a_{11}\right)-a_{12} a_{28}\right)\right.$

$+\frac{a_{12}}{2}\left(a_{19}\left(\theta-a_{11}\right)-a_{12} a_{29}\right)\left(\theta-a_{11}\right)^{2}$

$+a_{12}^{2}\left(\frac{a_{110}\left(\theta-a_{11}\right)}{2}-\frac{a_{12} a_{210}}{2}\right)\left(\theta-a_{11}\right)$

$\left.+\left(\frac{a_{111}\left(\theta-a_{11}\right)}{6}-\frac{a_{12} a_{211}}{6}\right)\left(\theta-a_{11}\right)^{3}\right) \widetilde{w}^{3}$

$+\left(\frac{a_{12}}{2}\left(a_{19}\left(\theta-a_{11}\right)-a_{12} a_{29}\right) \varphi^{2}\right.$

$\left.+\left(\frac{a_{111}\left(\theta-a_{11}\right)}{2}-\frac{a_{12} a_{211}}{6}\right)\left(\theta-a_{11}\right) \varphi^{2}\right) \widetilde{w} \widetilde{z}^{2}$

$-\left(a_{12}\left(a_{19}\left(\theta-a_{11}\right)-a_{12} a_{29}\right)\left(\theta-a_{11}\right) \varphi\right.$

$+a_{12}^{2}\left(\frac{a_{110}\left(\theta-a_{11}\right)}{2}-\frac{a_{12} a_{210}}{2}\right) \varphi$

$\left.+\left(\frac{a_{111}\left(\theta-a_{11}\right)}{2}-\frac{a_{12} a_{211}}{2}\right)\left(\theta-a_{11}\right)^{2} \varphi\right) \widetilde{w}^{2} \widetilde{z}$

$-\left(\frac{a_{111}\left(\theta-a_{11}\right)}{6}-\frac{a_{12} a_{211}}{6}\right) \varphi^{3} \widetilde{z}^{3}$

$+o\left((|\widetilde{w}|+|\widetilde{z}|)^{4}\right)$.

Calculating the two- and three-order derivatives of $G_{1}(\widetilde{w}, \widetilde{z})$ and $G_{2}(\widetilde{w}, \widetilde{z})$ at $\widetilde{w}=0$ and $\widetilde{\tau}=0$, the following results are obtained:

$$
\begin{aligned}
G_{1 \widetilde{w} \widetilde{w}} & =\frac{a_{12}^{2} a_{13}}{2} \varphi+\frac{a_{14}}{2} \varphi\left(\theta-a_{11}\right)^{2}+a_{12} a_{15}\left(\theta-a_{11}\right) \\
\cdot \varphi & , \\
G_{1 \widetilde{w z}} & =-\left(a_{14}\left(\theta-a_{11}\right) \varphi^{2}+a_{12} a_{15} \varphi^{2}\right), \\
G_{1 \tilde{z} \tilde{z}} & =\frac{a_{14}}{2} \varphi^{3},
\end{aligned}
$$




$$
\begin{aligned}
& G_{1 \widetilde{w} \widetilde{w}}=\frac{a_{12}^{3} a_{18}}{6}+\frac{a_{12} a_{19}}{2} \varphi\left(\theta-a_{11}\right)^{2}+\frac{a_{12}^{2} a_{110}}{2} \varphi(\theta \\
& \left.-a_{11}\right)+\frac{1}{6} a_{111} \varphi\left(\theta-a_{11}\right)^{3} \text {, } \\
& G_{1 \widetilde{w} \widetilde{w}}=-\left(a_{12} a_{19}\left(\theta-a_{11}\right) \varphi^{2}+\frac{a_{12}^{2} a_{110}}{2} \varphi^{2}\right. \\
& \left.+\frac{a_{111}}{2}\left(\theta-a_{11}\right)^{2} \varphi^{2}\right) \\
& G_{1 \widetilde{w} \tilde{z} \tilde{z}}=\frac{a_{12} a_{19}}{2} \varphi^{3}+\frac{a_{111}}{2} \varphi^{3}\left(\theta-a_{11}\right), \\
& G_{1 \tilde{z} \tilde{z} \tilde{z}}=-\frac{a_{111}}{6} \varphi^{4}, \\
& G_{2 \widetilde{w} \widetilde{w}}=\frac{a_{12}^{2}}{2}\left(a_{13}\left(\theta-a_{11}\right)-a_{12} a_{23}\right) \\
& +\frac{1}{2}\left(a_{14}\left(\theta-a_{11}\right)-a_{12} a_{24}\right)\left(\theta-a_{11}\right)^{2}+a_{12}(\theta \\
& \left.-a_{11}\right)\left(a_{15}\left(\theta-a_{11}\right)-a_{12} a_{25}\right), \\
& G_{2 \widetilde{w z}}=-\left(\left(a_{14}\left(\theta-a_{11}\right)-a_{12} a_{24}\right)\left(\theta-a_{11}\right) \varphi\right. \\
& \left.+a_{12}\left(a_{15}\left(\theta-a_{11}\right)-a_{12} a_{25}\right) \varphi\right), \\
& G_{2 \tilde{z} \tilde{z}}=\left(\frac{a_{14}\left(\theta-a_{11}\right)}{2}-\frac{a_{12} a_{24}}{2}\right) \varphi^{2}, \\
& G_{2 \widetilde{w} \widetilde{w}}=a_{12}^{3}\left(\frac{a_{18}\left(\theta-a_{11}\right)}{6}-\frac{a_{12} a_{28}}{6}\right) \\
& +a_{12}\left(\frac{a_{19}\left(\theta-a_{11}\right)}{2}-\frac{a_{12} a_{29}}{2}\right)\left(\theta-a_{11}\right)^{2} \\
& +a_{12}^{2}\left(\frac{a_{110}\left(\theta-a_{11}\right)}{2}-\frac{a_{12} a_{210}}{2}\right)\left(\theta-a_{11}\right) \\
& +\left(\frac{a_{111}\left(\theta-a_{11}\right)}{6}-\frac{a_{12} a_{211}}{6}\right)\left(\theta-a_{11}\right)^{3}, \\
& G_{2 \widetilde{w} \widetilde{w}}=-\left(a_{12}\left(a_{19}\left(\theta-a_{11}\right)-a_{12} a_{29}\right)\left(\theta-a_{11}\right) \varphi\right. \\
& +a_{12}^{2}\left(\frac{a_{110}\left(\theta-a_{11}\right)}{2}-\frac{a_{12} a_{210}}{2}\right) \varphi \\
& \left.+\left(\frac{a_{111}\left(\theta-a_{11}\right)}{2}-\frac{a_{12} a_{211}}{2}\right)\left(\theta-a_{11}\right)^{2} \varphi\right), \\
& G_{2 \widetilde{w} \tilde{z}}=\frac{a_{12}}{2}\left(a_{19}\left(\theta-a_{11}\right)-a_{12} a_{29}\right) \varphi^{2} \\
& +\left(\frac{a_{111}\left(\theta-a_{11}\right)}{2}-\frac{a_{12} a_{211}}{2}\right)\left(\alpha-a_{11}\right) \varphi^{2}, \\
& G_{2 \widetilde{z} \tilde{z} \tilde{z}}=-\left(\frac{a_{111}\left(\theta-a_{11}\right)}{6}-\frac{a_{12} a_{211}}{6}\right) \varphi^{3} .
\end{aligned}
$$

On the basis of map (41), the predator-prey system experiencing Neimark-Sacker bifurcation needs the requirement of the following condition:

$$
\begin{aligned}
a_{0}= & -\operatorname{Re}\left(\frac{(1-2 \bar{\lambda}) \bar{\lambda}^{2}}{1-\lambda} \xi_{11} \xi_{20}\right)-\frac{1}{2}\left|\xi_{11}\right|^{2}-\left|\xi_{02}\right|^{2} \\
& +\operatorname{Re}\left(\bar{\lambda} \xi_{21}\right) \neq 0,
\end{aligned}
$$

where

$$
\begin{aligned}
& \xi_{20}=\frac{1}{8}\left(\left(G_{1 \widetilde{w} \widetilde{w}}-G_{1 \tilde{z} \tilde{z}}+2 G_{2 \widetilde{w z}}\right)\right. \\
& \left.+\widetilde{i}\left(G_{2 \widetilde{w} \widetilde{w}}-G_{2 \tilde{z} \widetilde{z}}-2 G_{1 \widetilde{w} z}\right)\right), \\
& \xi_{11}=\frac{1}{4}\left(\left(G_{1 \widetilde{w} \widetilde{w}}+G_{1 \tilde{z} \widetilde{z}}\right)+\widetilde{i}\left(G_{2 \widetilde{w} \widetilde{w}}+G_{2 \widetilde{z} \widetilde{z}}\right)\right), \\
& \xi_{02}=\frac{1}{8}\left(\left(G_{1 \widetilde{w} \widetilde{w}}-G_{1 \tilde{z} \tilde{z}}-2 G_{2 \widetilde{w z}}\right)\right. \\
& \left.+\tilde{i}\left(G_{2 \widetilde{w} \widetilde{w}}-G_{2 \tilde{z} \widetilde{z}}+2 G_{1 \widetilde{w} \tilde{z}}\right)\right), \\
& \xi_{21}=\frac{1}{16}\left(\left(G_{1 \widetilde{w} \widetilde{w}}+G_{1 \widetilde{w z} \tilde{z}}+G_{2 \widetilde{w} \widetilde{w}}+G_{2 \widetilde{z} \widetilde{z}}\right)\right. \\
& \left.+\widetilde{i}\left(G_{2 \widetilde{w} \widetilde{w}}+G_{2 \widetilde{w} \tilde{z}}-G_{1 \widetilde{w} \widetilde{z}}-G_{1 \widetilde{z} \widetilde{z}}\right)\right) .
\end{aligned}
$$

Using the above formula, we can get

$$
\begin{aligned}
& a_{0}=-\frac{1}{A_{0}}\left\{M _ { 0 } \left[\left(G_{1 \widetilde{w} \widetilde{w}}+G_{1 \tilde{z} \tilde{z}}\right)\right.\right. \\
& \cdot\left(G_{1 \widetilde{w} \widetilde{w}}-G_{1 \tilde{z} \tilde{z}}+2 G_{2 \widetilde{w}}\right)-\left(G_{2 \widetilde{w} \widetilde{w}}+G_{2 \widetilde{z} \tilde{z}}\right) \\
& \left.\cdot\left(G_{2 \widetilde{w} \widetilde{w}}-G_{2 \tilde{z} \tilde{z}}-2 G_{1 \widetilde{w}}\right)\right]+N_{0}\left[\left(G_{2 \widetilde{w} \widetilde{w}}+G_{2 \tilde{z} \tilde{z}}\right)\right. \\
& \cdot\left(G_{1 \widetilde{w} \widetilde{w}}-G_{1 \tilde{z} \tilde{z}}+2 G_{2 \widetilde{w} \tilde{z}}\right)-\left(G_{1 \widetilde{w} \widetilde{w}}+G_{1 \tilde{z} \tilde{z}}\right) \\
& \left.\left.\cdot\left(G_{2 \widetilde{w} \widetilde{w}}-G_{2 \widetilde{z} \tilde{z}}-2 G_{1 \widetilde{w} \tilde{z}}\right)\right]\right\}-\frac{1}{32 a_{12}^{2} \varphi^{2}}\left[\left(G_{1 \widetilde{w} \widetilde{w}}\right.\right. \\
& \left.\left.+G_{1 \tilde{z} \widetilde{z}}\right)^{2}+\left(G_{2 \widetilde{w} \widetilde{w}}+G_{2 \tilde{z} \tilde{z}}\right)^{2}\right]-\frac{1}{64 a_{12}^{2} \varphi^{2}}\left[\left(G_{1 \widetilde{w} \widetilde{w}}\right.\right. \\
& \left.\left.-G_{1 \tilde{z} \widetilde{z}}-2 G_{2 \widetilde{w} \widetilde{z}}\right)^{2}+\left(G_{2 \widetilde{w} \widetilde{w}}-G_{2 \widetilde{z} \tilde{z}}+2 G_{1 \widetilde{w z}}\right)^{2}\right] \\
& +\frac{1}{16 a_{12} \varphi}\left[\alpha\left(G_{1 \widetilde{w} \widetilde{w}}+G_{1 \widetilde{w} \tilde{z}}+G_{2 \widetilde{w} \widetilde{z}}+G_{2 \tilde{z} \tilde{z} \tilde{z}}\right)\right.
\end{aligned}
$$

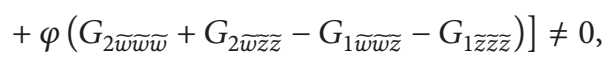

in which

$$
\begin{aligned}
A_{0}= & 32 a_{12}^{2} \varphi^{2}\left((1-\theta)^{2}+\varphi^{2}\right), \\
N_{0}= & 2 \theta \varphi\left((1-\theta)(1-2 \theta)-2 \varphi^{2}\right) \\
& -\varphi(3-4 \theta)\left(\theta^{2}-\varphi^{2}\right), \\
M_{0}= & \left(1-3 \theta+2 \theta^{2}-2 \varphi^{2}\right)\left(\theta^{2}-\varphi^{2}\right) \\
& +\left(6 \theta-8 \theta^{2}\right) \varphi^{2} .
\end{aligned}
$$




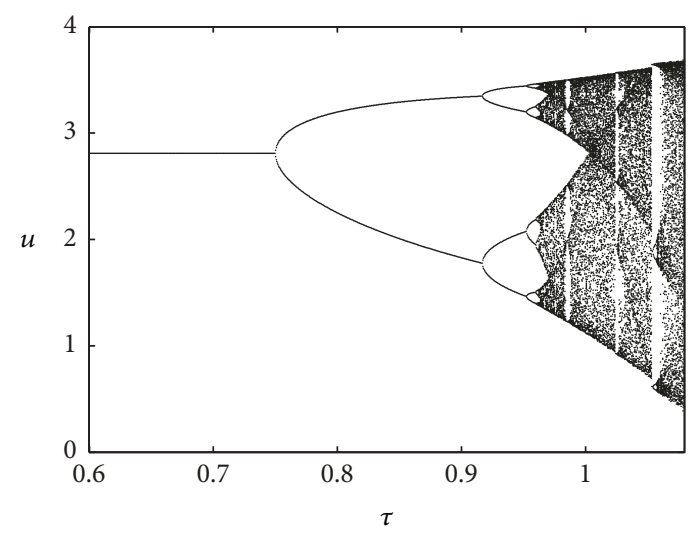

(a)

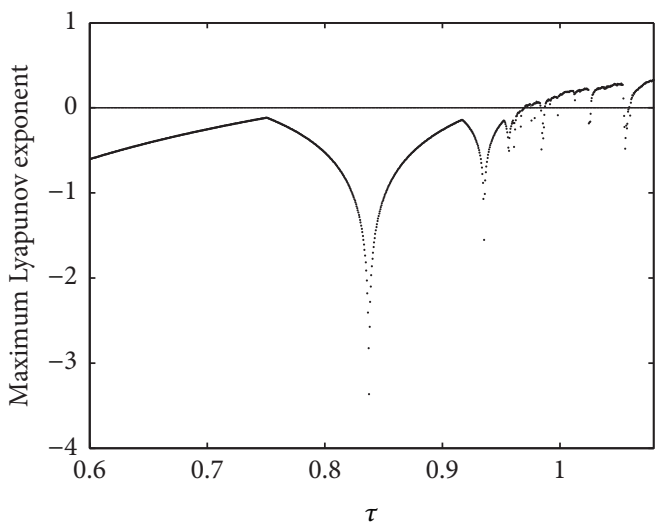

(b)

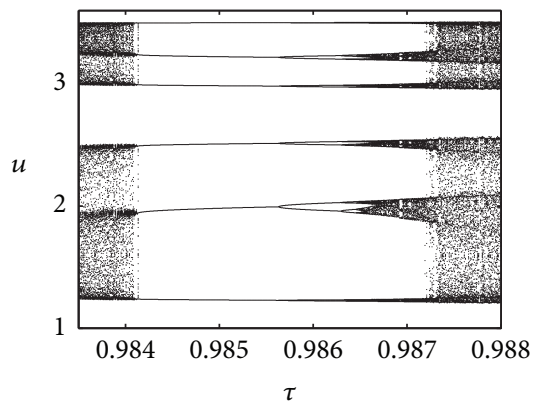

(c)

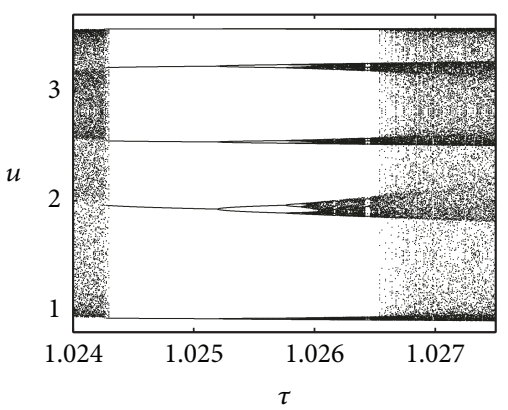

(d)

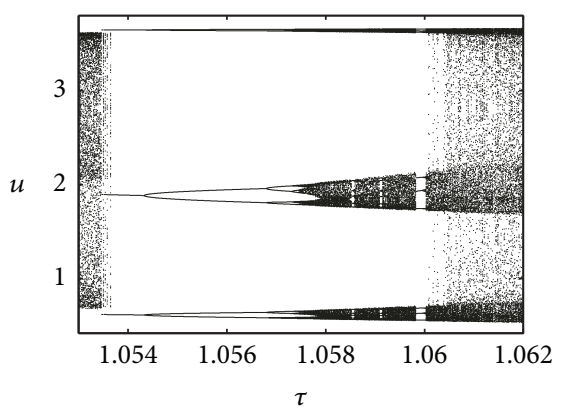

(e)

FIGURE 1: (a) Flip bifurcation diagram with $\tau$ covering [0.6, 1.08]; (b) maximum Lyapunov exponent corresponding to (a); (c)-(e) local amplifications of (a) showing periodic windows. $a=3, b=2, c=0.2, d=1, \alpha=1$, and $\beta=2$.

Taking the above calculations together, the occurrence of Neimark-Sacker bifurcation at the fixed point $\left(u_{2}, v_{2}\right)$ in the discrete predator-prey system needs the satisfaction of conditions (34), (39), and (46). Moreover, if $a_{0}<0$ and $\gamma>0$, an attracting invariant closed curve bifurcates from the fixed point for $\tau>\tau_{0}$; if $a_{0}>0$ and $\gamma>0$, a repelling invariant closed curve bifurcates from the fixed point for $\tau<\tau_{0}$.

\section{Numerical Results}

To show the bifurcations and complex dynamics of the discrete predator-prey system, numerical simulations are carried out. We fix the parameters as $a=3, b=2, c=0.2$, $d=1, \alpha=1$, and $\beta=2$ and assume that $\tau$ varies. Under such conditions, the fixed point is $(2.8086,1.3436)$ and the critical point for flip bifurcation is $\tau^{*}=0.7501$. At the critical bifurcation point, the two eigenvalues are $\lambda_{1}=-1$ and $\lambda_{2}=0.8899$. Considering the dynamics of map (23) restricted to the center manifold, we then obtain the values of two quantities as $\eta_{1}=-2.6665$ and $\eta_{2}=-0.0036$. Therefore, the predator-prey system indeed undergoes flip bifurcation at $\left(u_{2}, v_{2}\right)$ in a small neighborhood of $\tau=\tau^{*}$, and the system dynamics converge to a period-2 orbit. Taking $\tau=0.8$ as an example, the two stable periodic points are $(3.1929,1.1228)$ and $(2.2583,1.094)$.

Figure 1(a) displays the flip bifurcation diagram of the discrete predator-prey system with $\tau$ varying in $[0.6,1.08]$. It shows a period-doubling cascade in orbits of periods $2,4,8$, and 16 (Figures 2(a)-2(d)). As determined by the maximum Lyapunov exponent in Figure 1(b), we find that the system firstly experiences chaos at $\tau=0.9698$. Figures $1(\mathrm{c})-1(\mathrm{e})$ are local amplifications of the bifurcation diagram, displaying three periodic windows occurring in the chaotic region. In these periodic windows, period-6, period-5, and period-3 orbits appear (Figures 2(g), 2(j), and 2(m)). Moreover, a subperiod-doubling cascade emerges in each periodic window, leading to interior chaotic crisis and dynamic transition from periodic behaviors to chaos (Figures $2(\mathrm{~m})-2(\mathrm{p})$ ). Figure 2 shows various attractors in the flip bifurcation diagram with the increase of $\tau$ value.

Figure 3 displays Neimark-Sacker bifurcation of the discrete predator-prey system with parametric conditions given as $a=2, b=2, c=2, d=1.75, \alpha=0.1$, and $\beta=0.1$. In this case, the Neimark-Sacker bifurcation emerges at the fixed point $(1.3467,0.3849)$ in a small neighborhood of $\tau^{*}=$ 1.1166. We can verify that, at the critical bifurcation point, $\lambda_{1,2}=0.2500 \pm 0.9682 \tilde{i}$ and $\left|\lambda_{1,2}\right|=1$. The values of $\gamma$ and $a_{0}$ are determined as $\gamma=0.6716$ and $a_{0}=-1.4078$. Since $\gamma>0$ and $a<0$, we know that an attracting invariant closed curve bifurcates from the fixed point for $\tau>\tau_{0}$.

The bifurcation diagram in Figure 3(a) and the maximum Lyapunov exponent diagram in Figure 3(b) suggest that the Neimark-Sacker bifurcation induces a route to chaos, leading to a dynamic transition from a fixed point, to invariant curves, with periodic windows occurring in between, to chaotic dynamics. The maximum Lyapunov exponent 


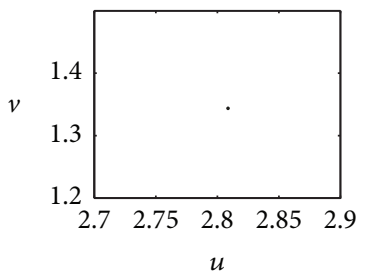

(a) $\tau=0.7$

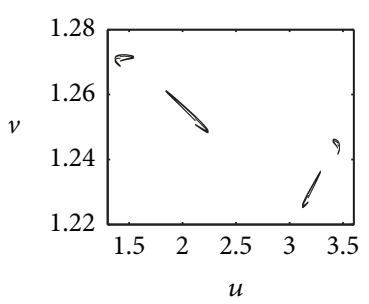

(e) $\tau=0.964$

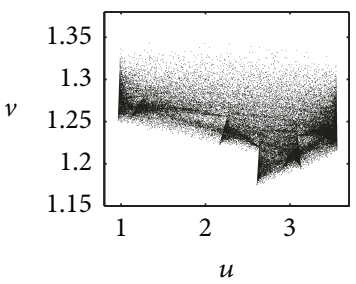

(i) $\tau=1.02$

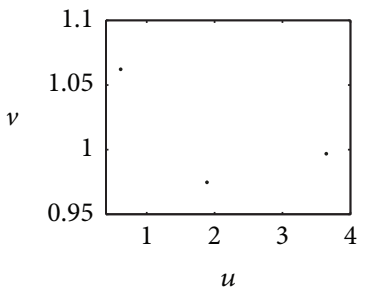

(m) $\tau=1.054$

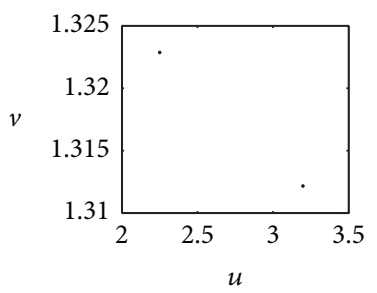

(b) $\tau=0.8$

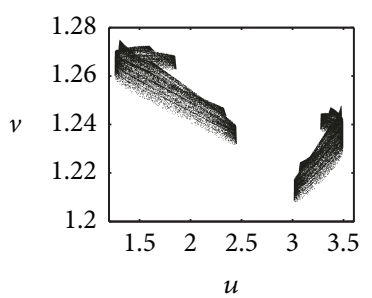

(f) $\tau=0.98$

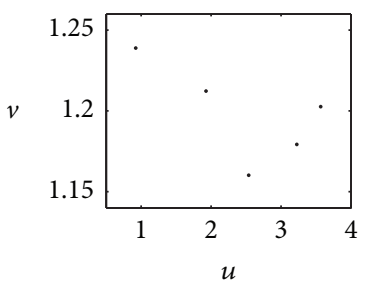

(j) $\tau=1.025$

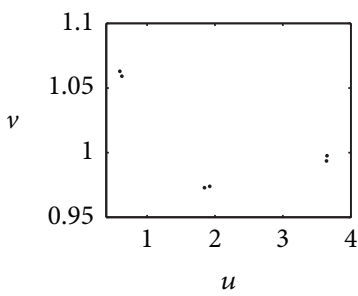

(n) $\tau=1.055$

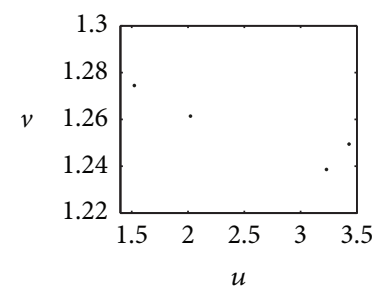

(c) $\tau=0.94$

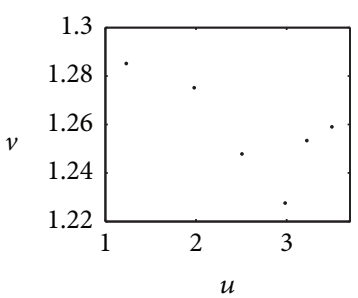

(g) $\tau=0.985$

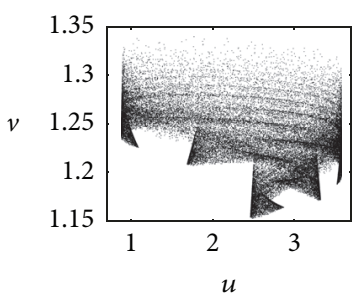

(k) $\tau=1.03$

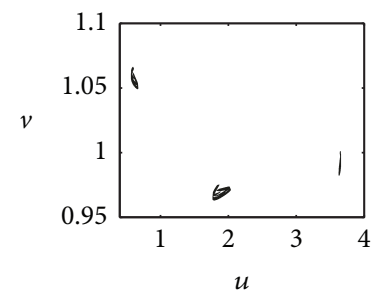

(o) $\tau=1.058$

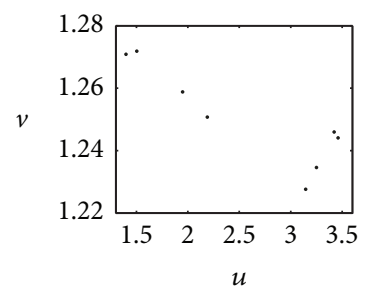

(d) $\tau=0.96$

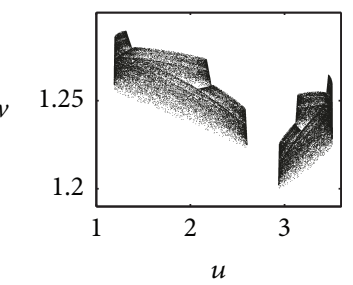

(h) $\tau=0.99$

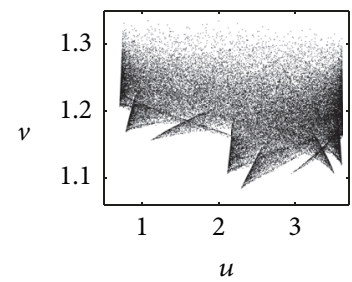

(l) $\tau=1.05$

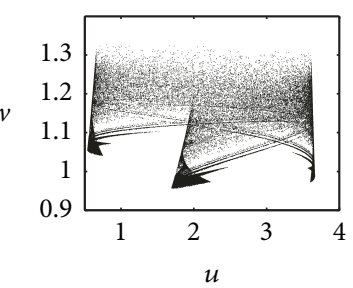

(p) $\tau=1.061$

FIGURE 2: Phase portraits for various values of $\tau$ corresponding to Figure 1(a).

diagram says that the predator-prey system firstly shows chaotic dynamics at $\tau=1.4072$. Local amplifications in Figures 3(c)-3(h) illustrate that the invariant curves may show many isolated cycles and periodic windows can appear in the zones of both invariant curves and chaos. In the invariant curve zone, the periodic windows often emerge intermittently, whereas in the chaotic zone, the periodic windows often exhibit sub-period-doubling cascade. In the periodic windows of Figures 3(c)-3(h), we find period-9, period-13, period-20, period-22 orbits. Figure 4 explicitly displays the alternation between periodic behaviors and invariant curves or chaotic dynamics with the increase of $\tau$ value.

Phase portraits for various values of $\tau$ corresponding to Figure 3(a) are shown in Figure 4. It is worth noting that Figure 4(1) is a chaotic attractor in the sense of Marotto. According to the definition of snap-back repeller and Marotto chaos [32], we can prove that the fixed point $\left(u_{2}, v_{2}\right)$ is a snapback repeller by an iterative method and the emergence of Marotto chaos. In the case of $a=2, b=2, c=2, d=1.75$, $\alpha=0.1, \beta=0.1$, and $\tau=1.48$, we have $\lambda_{1,2}=0.006 \pm 1.2833 \tilde{i}$ and $\left|\lambda_{1,2}\right|=1.2833>1$. Notice that the fixed point of $f$ with the norm of all eigenvalues of $D f(u, v)$ exceeding 1 in magnitude is equivalent to $1+p+q>0,1-p+q>0$, and $q>1$. Let $H_{1}, H_{2}$, and $H_{3}$ represent the sets of three inequalities in the front and $U r\left(u_{2}, v_{2}\right)=H^{1} \cap H^{2} \cap H^{3}$; then, we obtain the neighborhood $U r\left(u_{2}, v_{2}\right)=\{(u, v) \mid 1.04<u<1.53,0.21<$ $v<0.55\}$. We need to find a point $\left(u^{\prime}, v^{\prime}\right) \in U r\left(u_{2}, v_{2}\right)$, satisfying $\left(u^{\prime}, v^{\prime}\right)_{3}=\left(u_{2}, v_{2}\right)$ and $\operatorname{det}\left(D f\left(\left(u^{\prime}, v^{\prime}\right) k\right)\right) \neq 0$ for $1 \leqslant k \leqslant 3$, where $\left(u^{\prime}, v^{\prime}\right)_{k}=f^{k}\left(u^{\prime}, v^{\prime}\right)$. Through three iterative calculations and numerical simulations, we get the point $\left(u^{\prime}, v^{\prime}\right)=(1.1004,0.4816)$ which meets $f^{3}\left(u^{\prime}, v^{\prime}\right)=$ $\left(u_{2}, v_{2}\right)$ and $\operatorname{det}\left(D f\left(\left(u^{\prime}, v^{\prime}\right)_{k}\right)\right)=2.4284,1.3198,-2.5154 \neq 0$ for $1 \leqslant k \leqslant 3$. Through these calculations, we proved that $\left(u_{2}, v_{2}\right)$ is a snap-back repeller, and map (3) exhibits a chaotic behavior in the sense of Marotto.

With the variation of other parameter values (e.g., parameter $d$ ), the predator-prey system may exhibit richer dynamical behaviors in the Neimark-Sacker bifurcation diagram. When we set the parameter values as $a=2, b=2, c=2$, $d=1.85, \alpha=0.1$, and $\beta=0.1$, a new Neimark-Sacker 


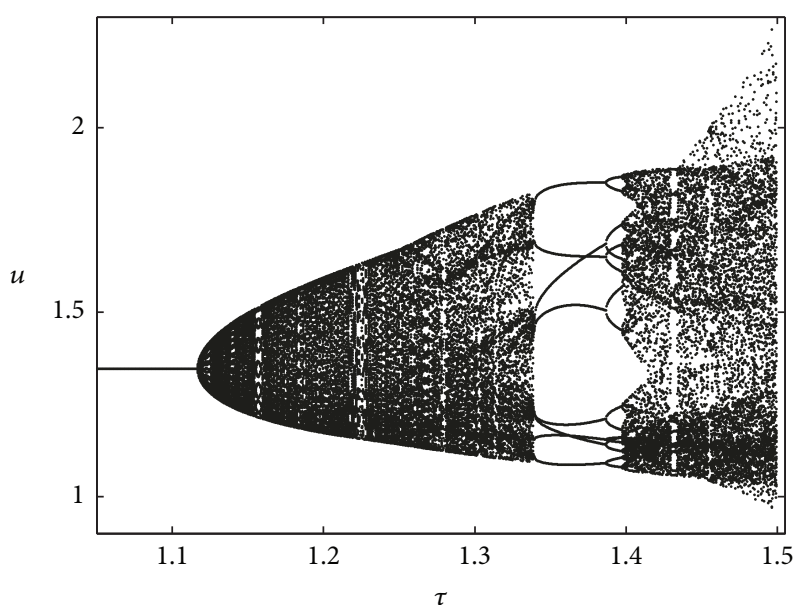

(a)

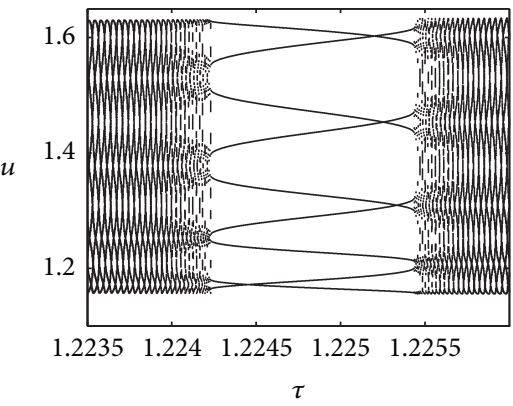

(c)

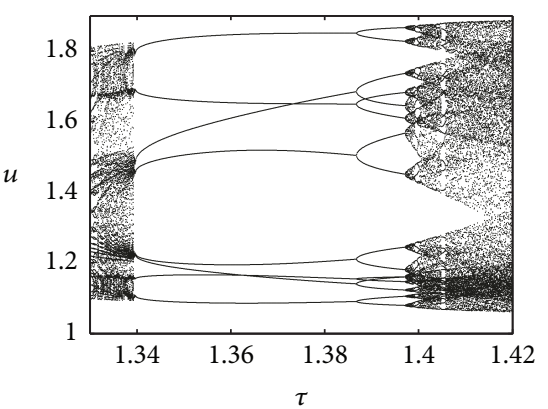

(f)

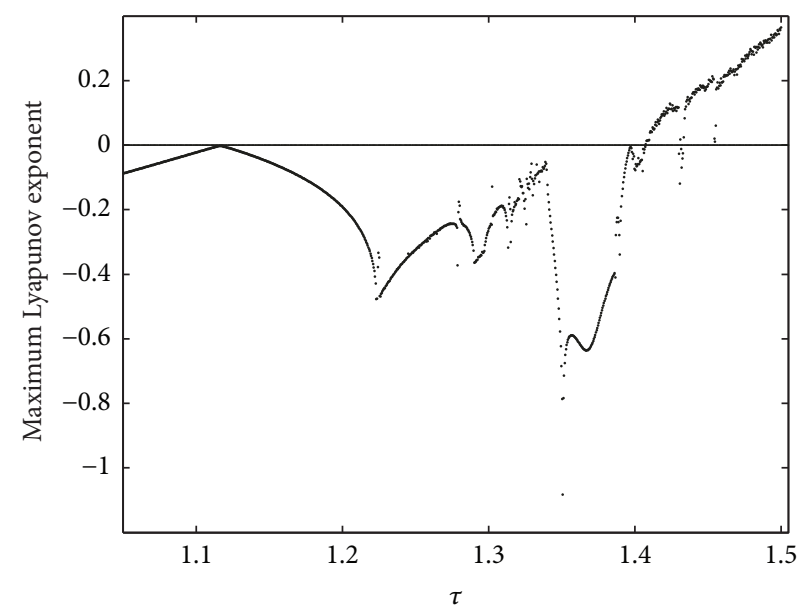

(b)

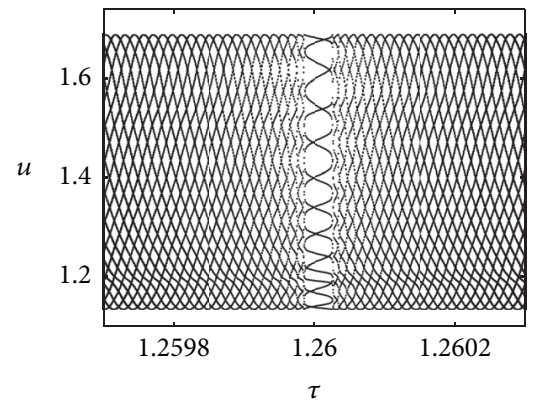

(d)

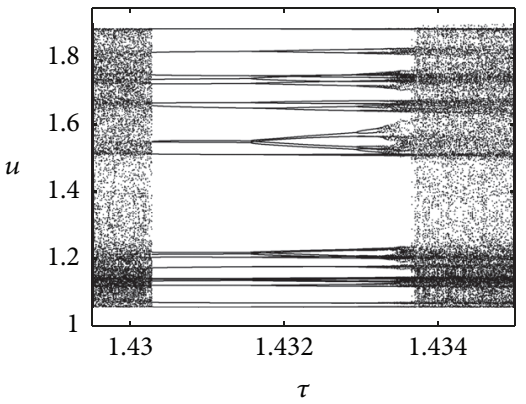

(g)

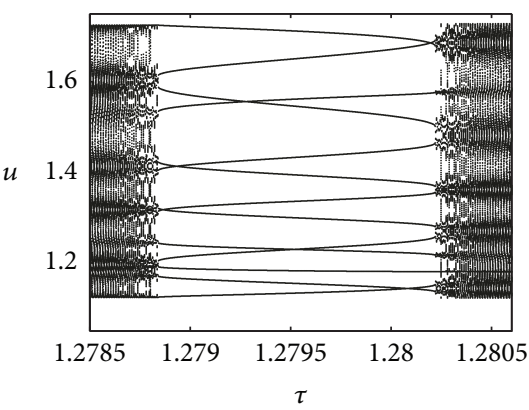

(e)

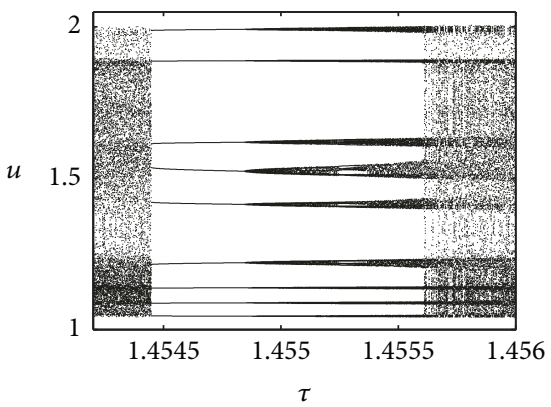

(h)

Figure 3: (a) Neimark-Sacker bifurcation diagram with $\tau$ covering [1.05, 1.505]; (b) maximum Lyapunov exponent corresponding to (a); (c)-(h) local amplifications of (a). $a=2, b=2, c=2, d=1.75, \alpha=0.1$, and $\beta=0.1$.

bifurcation diagram is obtained as shown in Figure 5(a). The system undergoes Neimark-Sacker bifurcation at $\tau=0.9492$, and it firstly enters chaotic dynamics at about $\tau=1.1964$ (Figures 5(a) and 6(b)). Similar nonlinear characteristics to Figures 3 and 4 are found in this case, such as route to chaos, invariant curves, chaotic attractors, and periodic windows, which here exhibit period-5, period-9, period-24, and period-34 orbits. However, in the periodic window of [1.3134, 1.3189], different dynamical behaviors are observed (Figure 5(c)). At about $\tau=1.3134$, the chaotic dynamics of the predator-prey system suddenly disappear and the system dynamics jump to a periodic behavior with period 7 (Figures 6(a) and 6(b)). On each branch of the period-7 orbit, sub-Neimark-Sacker bifurcation occurs (Figure 5(d)). In the phase portrait, this reflects as each periodic point changing to a cycle (Figure 6(c)). The Neimark-Sacker bifurcation in each branch of period-7 orbit also triggers a route to chaos like Figure 3(a), leading to 7 small chaotic subsets. Chaotic interior crisis then takes place and leads to an abrupt transition from chaotic subsets to a narrow chaotic band (Figures 5(c) and 6(d)). The chaotic band is in the range $[1.317,1.3171]$, and after that, the system dynamics turn to be periodic again (see period-15 orbit as shown in Figure 6(e)). Similarly, on each branch of the periodic orbit, sub-flip bifurcation occurs and induces a period-doubling cascade (Figures 5(e) and 6(e)-6(g)). Chaotic subsets and interior crisis appear sequently, taking the system entering chaotic dynamics again (Figure 6(h)). 


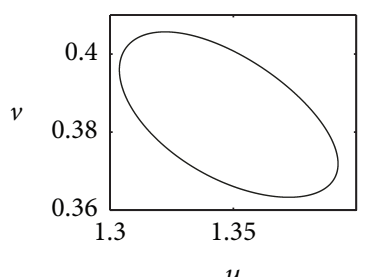

(a) $\tau=1.12$

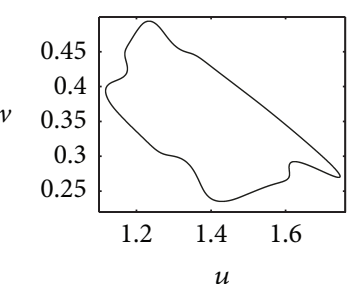

(e) $\tau=1.29$

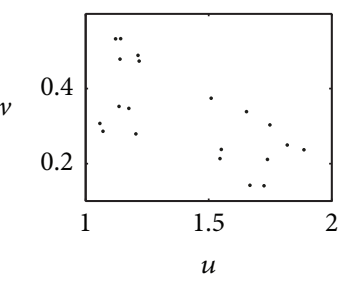

(i) $\tau=1.431$

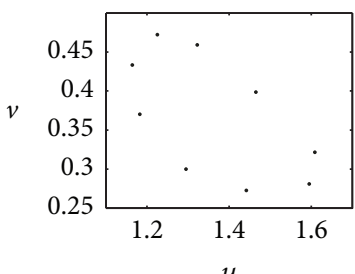

(b) $\tau=1.225$

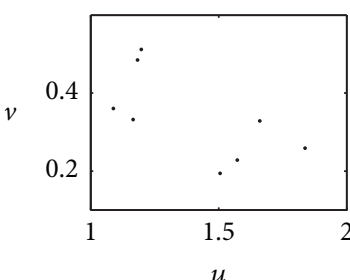

(f) $\tau=1.35$

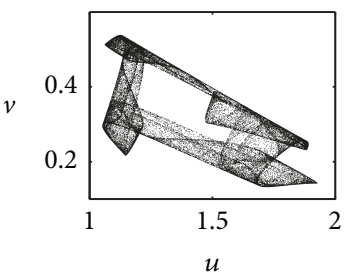

(j) $\tau=1.44$

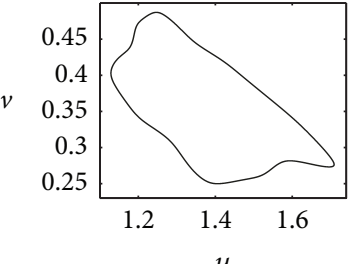

(c) $\tau=1.27$

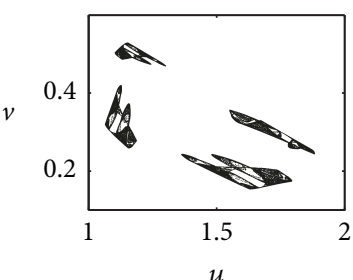

(g) $\tau=1.410$

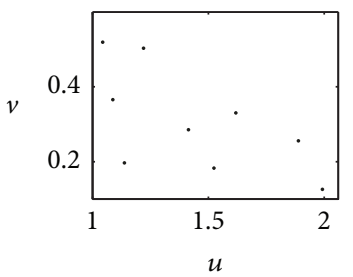

(k) $\tau=1.4548$

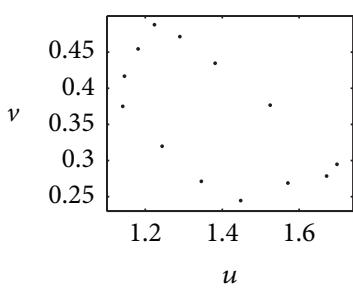

(d) $\tau=1.28$

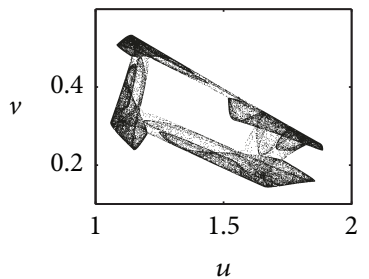

(h) $\tau=1.425$

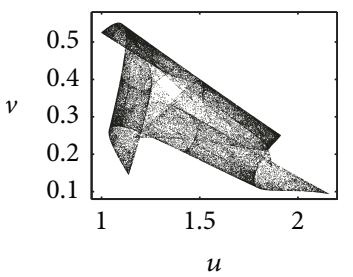

(l) $\tau=1.48$

FIgURE 4: Phase portraits for various values of $\tau$ corresponding to Figure 3(a).

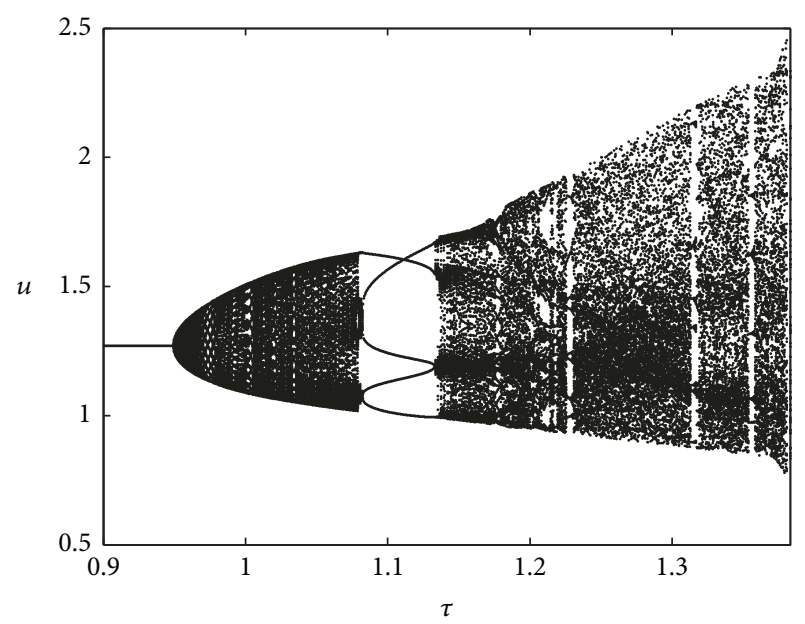

(a)

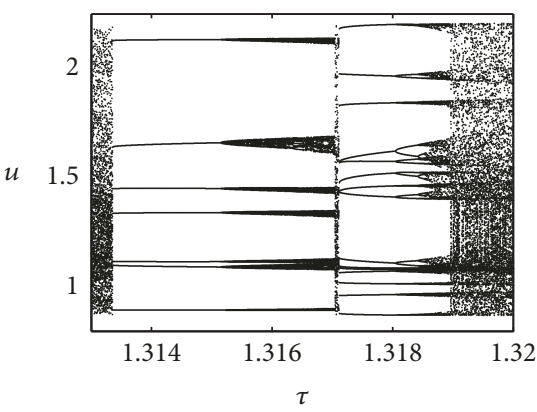

(c)

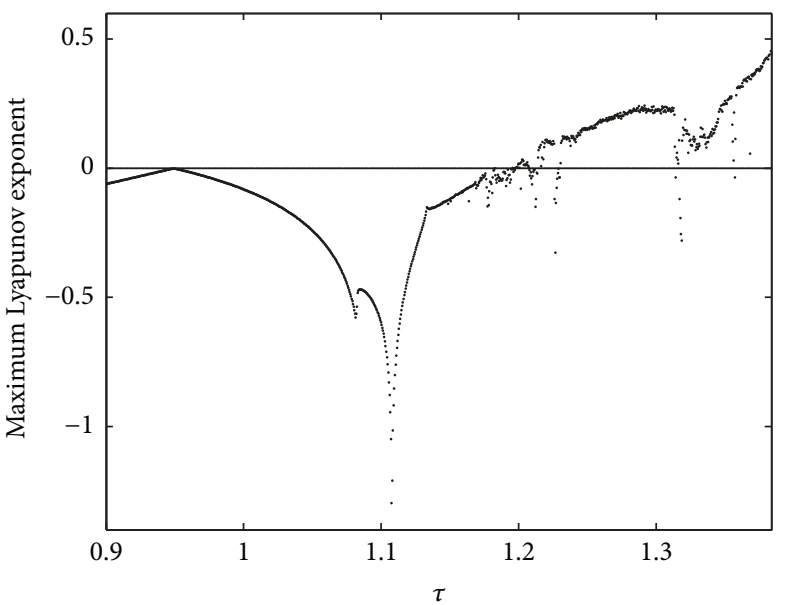

(b)

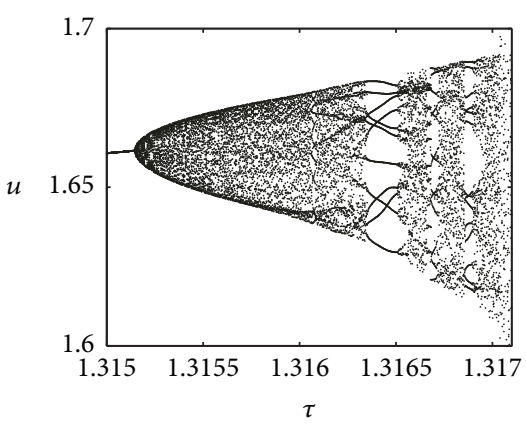

(d)

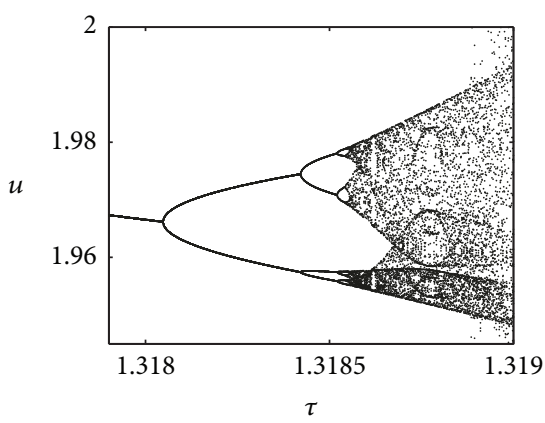

(e)

FIGURE 5: (a) Neimark-Sacker bifurcation diagram with parametric conditions given as $a=2, b=2, c=2, d=1.85, \alpha=0.1$, and $\beta=0.1$; (b) maximum Lyapunov exponent corresponding to (a); (c) local amplification of (a) in the range of [1.313, 1.32]; (d)-(e) amplifications of sub-Neimark-Sacker bifurcation and sub-flip bifurcation diagrams on each branch of periodic orbits. 


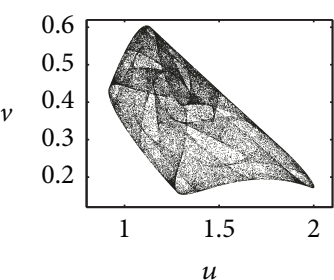

(a) $\tau=1.25$

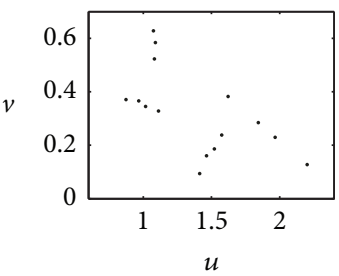

(e) $\tau=1.318$

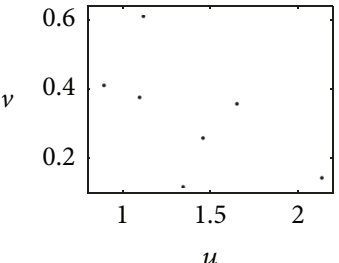

(b) $\tau=1.314$

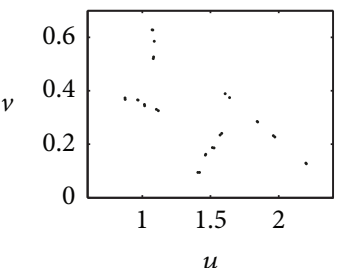

(f) $\tau=1.3182$

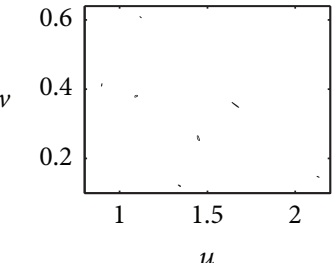

(c) $\tau=1.316$

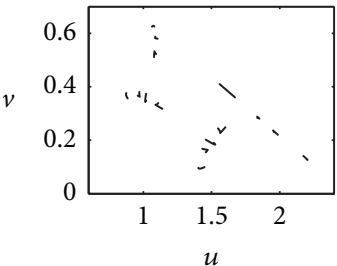

(g) $\tau=1.3188$

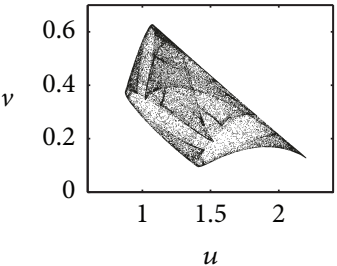

(d) $\tau=1.3171$

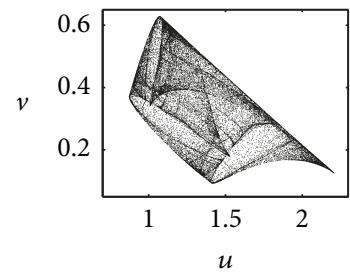

(h) $\tau=1.32$

FIgURE 6: Phase portraits for various values of $\tau$ corresponding to Figure 5(a).

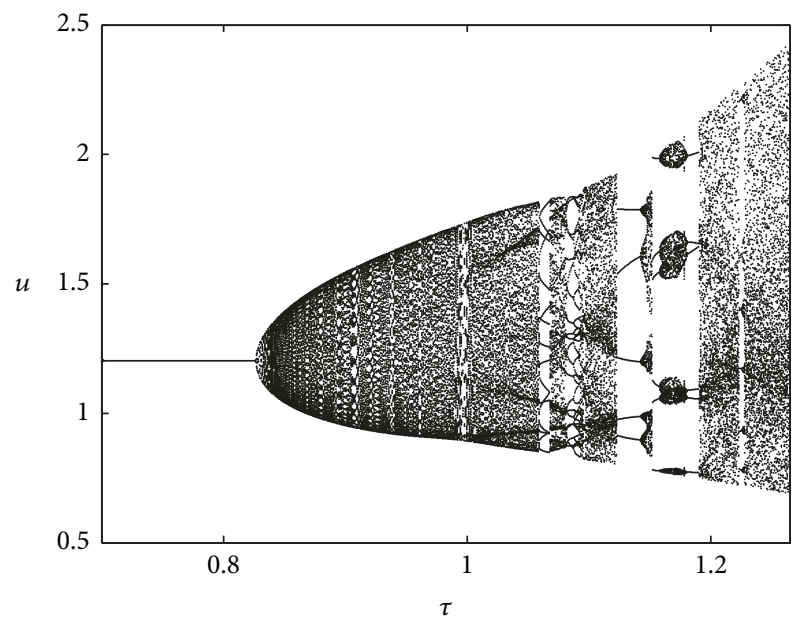

(a)

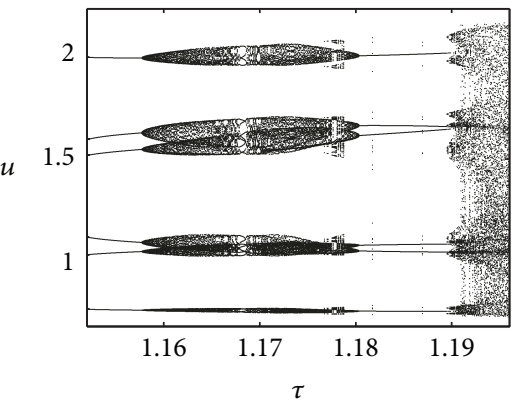

(c)

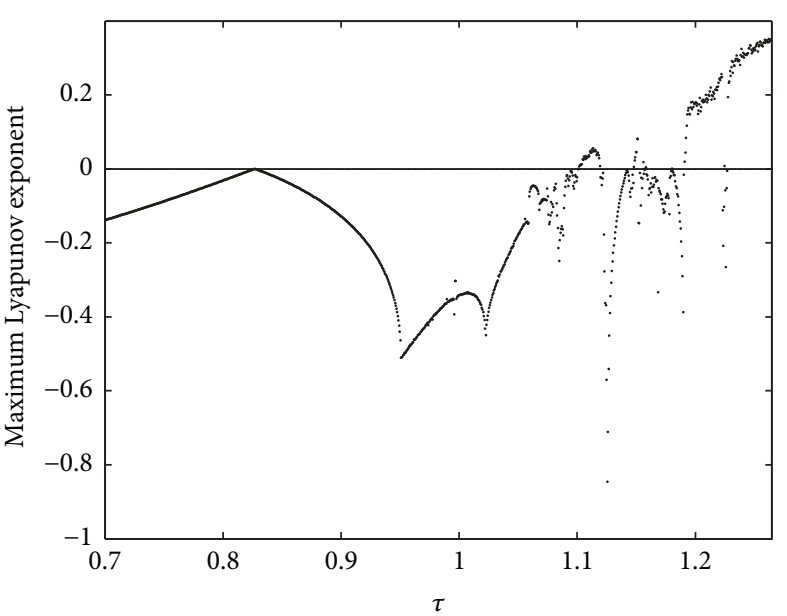

(b)

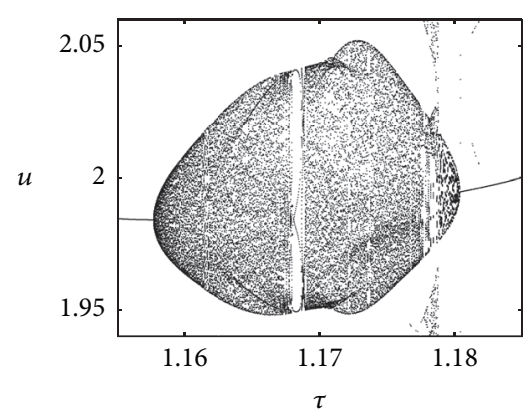

(d)

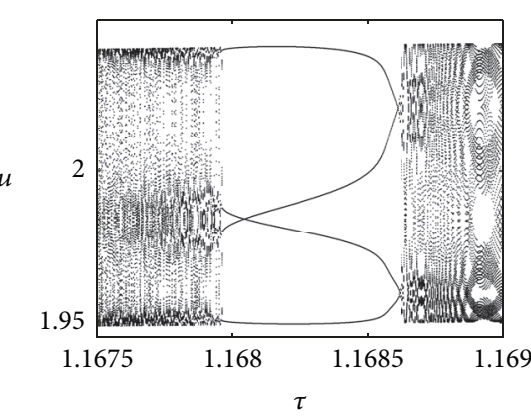

(e)

FiguRE 7: (a) Neimark-Sacker bifurcation diagram with parameter values given as $a=2, b=2, c=2, d=1.95, \alpha=0.1$, and $\beta=0.1$; (b) maximum Lyapunov exponent corresponding to (a); (c) local amplification of (a) in the range of [1.152, 1.196]; (d)-(e) amplifications of dynamics on each branch of period- 6 orbit in (c).

Figure 7 shows the Neimark-Sacker bifurcation diagram when the parameter values are given as $a=2, b=2$, $c=2, d=1.95, \alpha=0.1$, and $\beta=0.1$. The critical Neimark-Sacker bifurcation point is $\tau=0.9492$, and the first chaotic point is at around $\tau=1.1012$ (Figures $7(\mathrm{a})$ and $7(\mathrm{~b}))$. On the route to chaos, periodic windows with period-5, period-6, period-7, period-11, and period16 orbits and narrow chaotic band are found. When we amplify the range $[1.152,1.196]$, new dynamical behaviors are observed (Figure $7(\mathrm{c})$ ). On each branch of the period6 orbit, which occurs after the chaotic band (Figures 8(a) and $8(\mathrm{~b})$ ), the predator-prey system sequently undergoes 


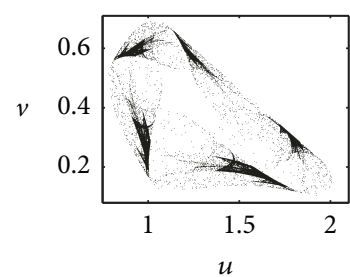

(a) $\tau=1.15$

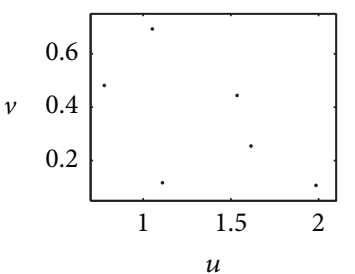

(b) $\tau=1.155$

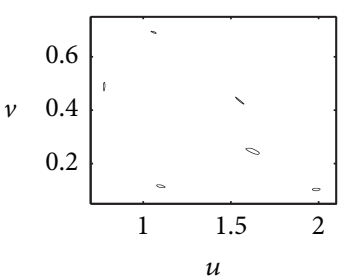

(c) $\tau=1.16$

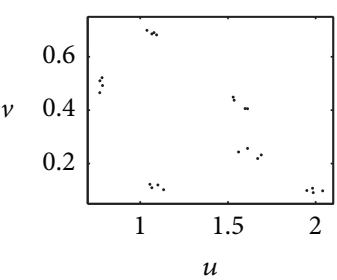

(d) $\tau=1.168$

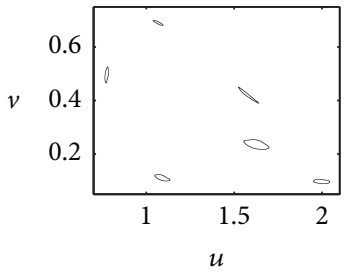

(e) $\tau=1.17$

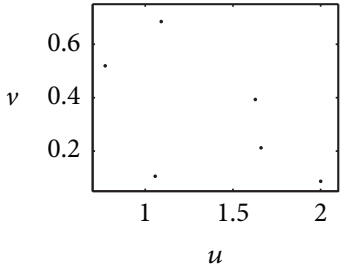

(f) $\tau=1.185$

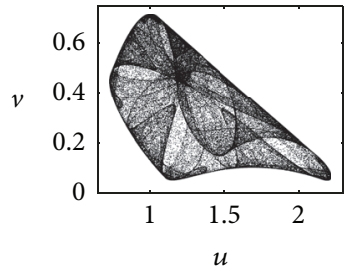

(g) $\tau=1.21$

FIGURE 8: Phase portraits for various values of $\tau$ corresponding to Figure 7(a).

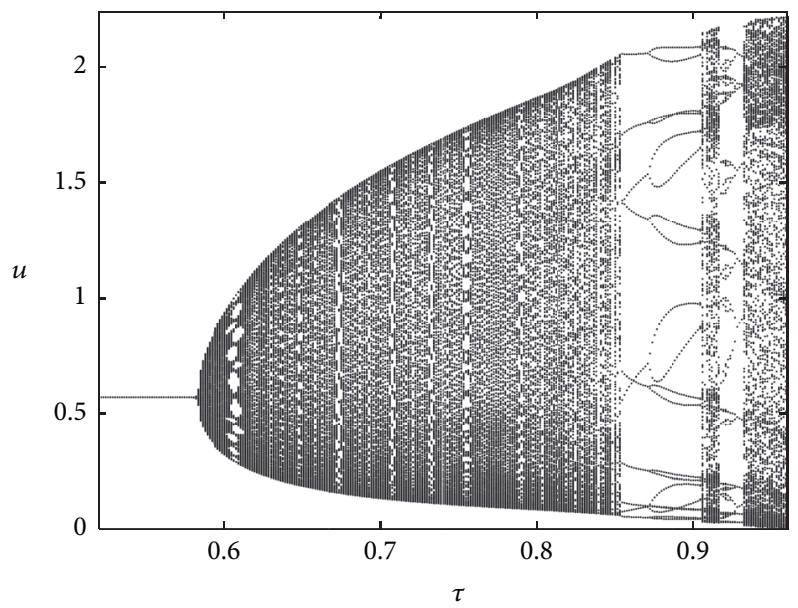

(a)

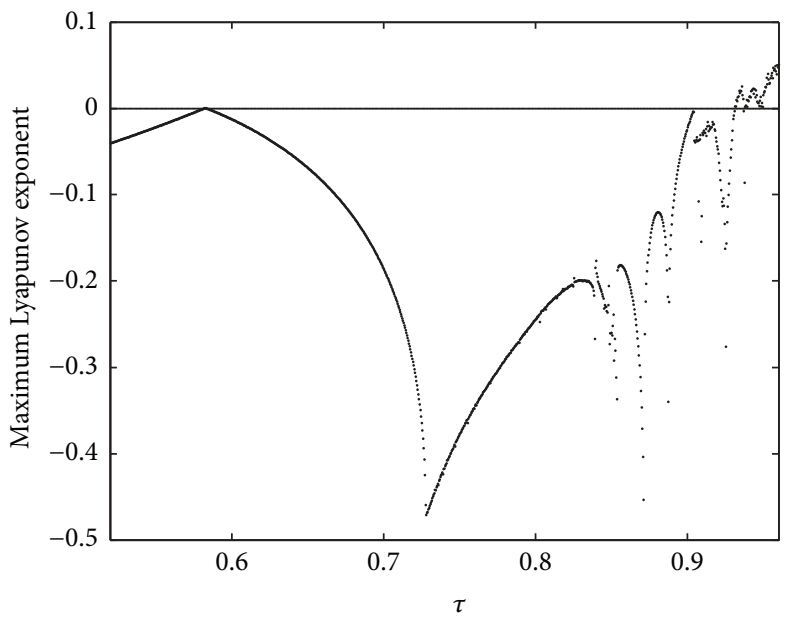

(b)

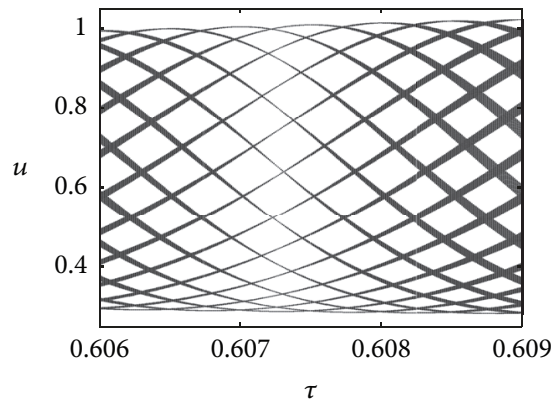

(c)

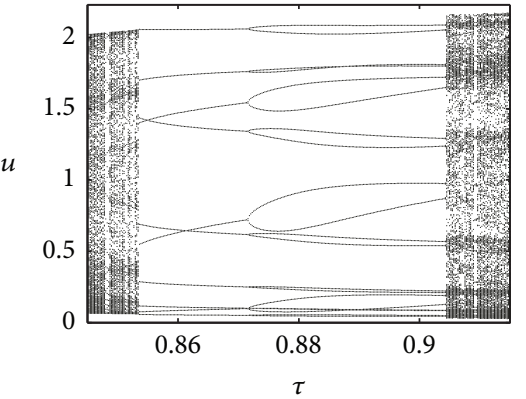

(d)

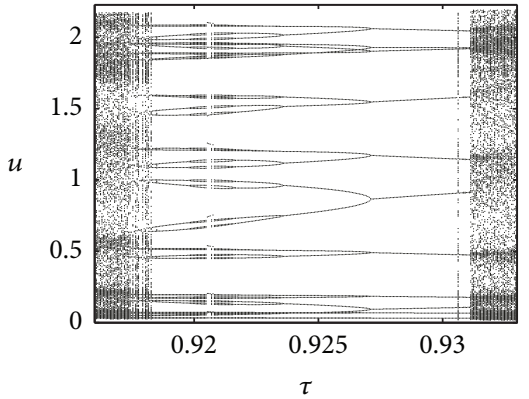

(e)

Figure 9: (a) Neimark-Sacker bifurcation diagram with parameter values given as $a=2, b=4, c=1, d=2.2, \alpha=0.05$, and $\beta=0.5$; (b) maximum Lyapunov exponent corresponding to (a); (c)-(e) local amplifications of (a) in the range of $[0.52,0.96]$.

sub-Neimark-Sacker bifurcation and periodic window and inverse sub-Neimark-Sacker bifurcation with the increase of $\tau$ value (Figures $7(\mathrm{~d})$ and $7(\mathrm{e})$ ). This process results in a dynamic transition from period-6 orbit, to invariant curves with 6 cycles, to period-24 orbit, and then the system experiences the above dynamical behaviors again but in an inverse way (Figures 8(b)-8(f)), until the emergence of chaotic behaviors (Figure $8(\mathrm{~g})$ ).

Figure 9 displays a case in which the bifurcation and route to chaos are restricted in the range of $0<\tau<1$. The parametric conditions for this case are given as $a=2$, $b=4, c=1, d=2.2, \alpha=0.05$, and $\beta=0.5$. The 


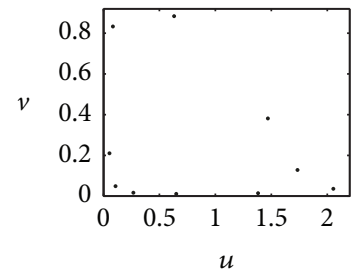

(a) $\tau=0.86$

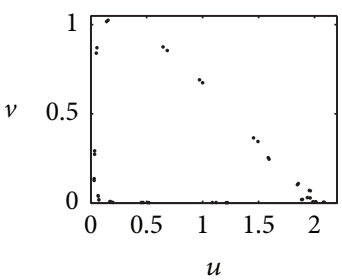

(e) $\tau=0.919$

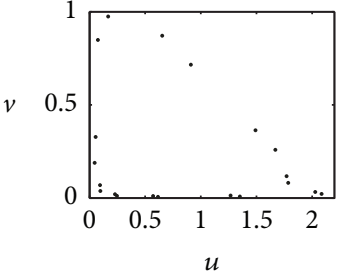

(b) $\tau=0.88$

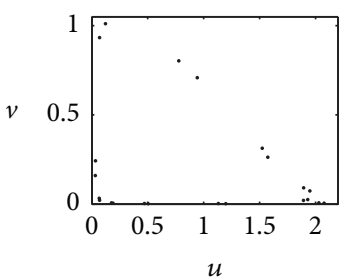

(f) $\tau=0.925$

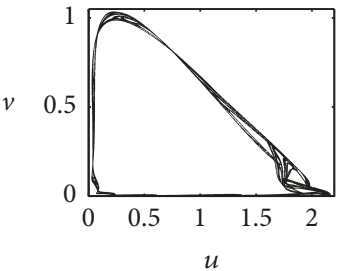

(c) $\tau=0.91$

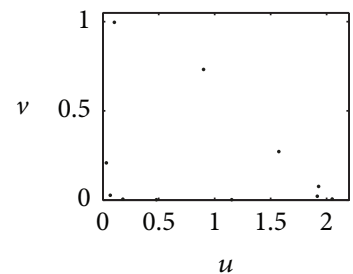

(g) $\tau=0.93$

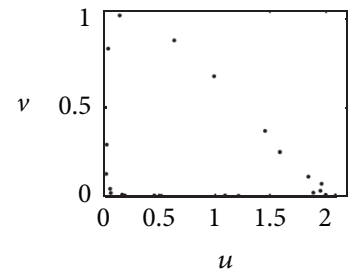

(d) $\tau=0.9175$

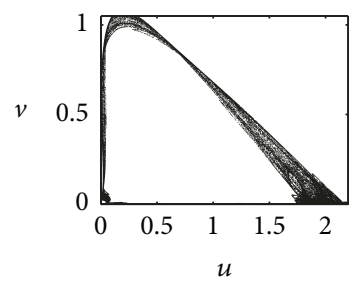

(h) $\tau=0.96$

FIGURE 10: Phase portraits for various values of $\tau$ corresponding to Figure 9(a).

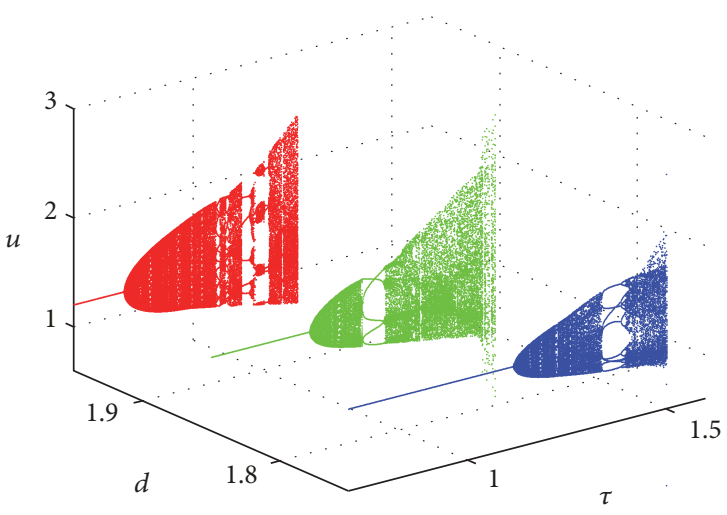

(a)

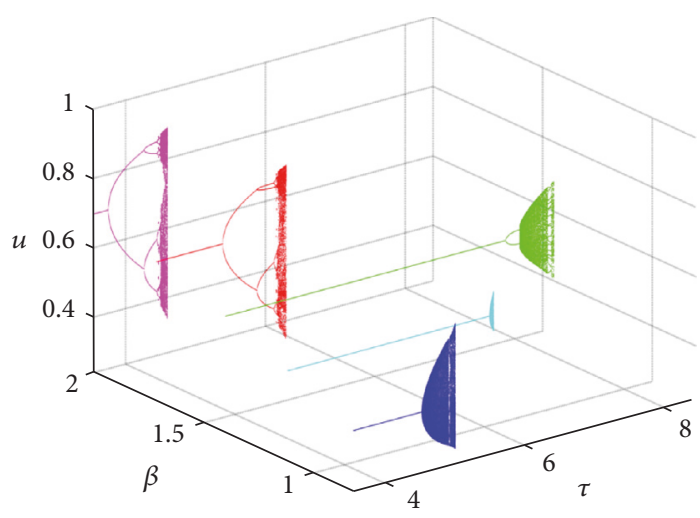

(b)

Figure 11: Bifurcation diagrams in $(\tau, d, u)$ space and $(\tau, \beta, u)$ space, describing (a) the change of Neimark-Sacker bifurcation when $a=2$, $b=2, c=2, \alpha=0.1, \beta=0.1$, and $d=1.75,1.85$, and 1.95 and (b) the transition between Neimark-Sacker bifurcation and flip bifurcation when $a=1, b=2, c=0.2, d=1, \alpha=1$, and $\beta=0.8,1.1,1.3851,1.7$, and 2 .

fixed point $(0.5727,0.4496)$ is a stable attractor at $\tau=0.55$. With the change of $\tau$ value, the fixed point loses its stability through Neimark-Sacker bifurcation at $\tau=0.5728$. The maximum Lyapunov exponent corresponding to Figure 9(a) is shown (Figure 9(b)); we find that the predator-prey system enters chaos at $\tau=0.9314$. Before the chaotic region, the predator-prey system undergoes a periodic window with period-10 orbits, and as the value of $\tau$ increases, the period orbit doubles (Figures 10(a) and 10(b)), causing the system to jump to quasiperiodic behaviors (Figure 9(d)), following which the system returns to another periodic window with period halving (Figures 9(e) and 10(d)-10(g)). And then, the system experiences intermittent chaos, which suggests the abrupt appearance of chaotic dynamics. The chaotic attractor is demonstrated by the cloud of points in Figure 10(h).

Figure 11 shows three-dimensional bifurcation diagrams in $(\tau, d, u)$ space and $(\tau, \beta, u)$ space with varying parameter values. As shown in Figure 11(a), we find that, with the increase of parameter $d$, the Neimark-Sacker bifurcation diagram moves toward the negative $\tau$ axis. Such movement takes the decrease of Neimark-Sacker bifurcation critical point and first chaotic point and simultaneously changes the periodic windows on the route to chaos. Figure 11(b) exhibits that, with the increase of parameter $\beta$, the predator-prey system presents a dynamic transition from Neimark-Sacker bifurcation to flip bifurcation. Moreover, we find a critical case between the two bifurcations, where the critical points of Neimark-Sacker bifurcation and flip bifurcation are the same and the two bifurcations combine together. With the given parametric conditions, we have $\tau^{*}=\tau_{0} \approx 1.3851$ in such case. The predator-prey system firstly experiences flip bifurcation and then abruptly jumps to the latter part of the NeimarkSacker bifurcation diagram.

\section{Conclusions}

In this research, the complex behaviors of discrete predatorprey systems with Crowley-Martin functional response are 
studied. Like many studies on the discrete predator-prey systems $[5,6,20,21,32]$, we found that the discrete system can undergo flip bifurcation and Neimark-Sacker bifurcation at the stably coexistent fixed point. The two bifurcations can both trigger the route to chaos; that is, chaotic dynamics appear along with the emergence of bifurcations. On the routes to chaos, we also find a period-doubling cascade in orbits of periods 2,4 , and 8 , periodic windows, invariant cycles, chaotic attractors, and Marotto chaos. Compared with the study of Ren et al. [32], who also investigated a discrete predator-prey system with Crowley-Martin functional response, many new interesting dynamic behaviors are revealed, including period-7, period-9, period-11, period-13, period-15, period-22, period-30, and period-34 orbits, sub(inverse) Neimark-Sacker bifurcation, chaotic interior crisis, chaotic band, and intermittent periodic behaviors. Moreover, in the three-dimensional bifurcation diagram, we find the transition between flip bifurcation and Neimark-Sacker bifurcation and a critical bifurcation case between the two bifurcations. These results indicate that the discrete predatorprey system has more complex and richer dynamics than the corresponding continuous system.

The generation of complex dynamics in the discrete predator-prey system is related to two important nonlinear mechanisms, flip bifurcation and Neimark-Sacker bifurcation, which cause the system to jump from steady state to oscillatory states, such as periodic and quasiperiodic states, and trigger routes to chaos. The occurrence of these behaviors induced by the two bifurcations can ecologically explain the complex dynamic states of populations. For example, the Australian entomologist Nicholson initiated a series of longterm experiments in which he kept populations of blowflies (Lucilia cuprina) in cages under a variety of resource renewal regimes. In one experiment, he found that the numbers of adult blowflies fluctuated as perturbed periodic state for the first 400 days [33]. Other experiments have investigated the long-term dynamics of freshwater organisms such as the cladoceran Daphnia in the laboratory. In one of the first such experiments, Slobodkin observed irregular fluctuations in time series that lasted between 200 and 400 days [34]. Application of the approaches for analyzing time series by computing Lyapunov exponents indicates that these time series may be quasiperiodic. Moreover, chaotic dynamics were also found in the natural world or laboratory experiments. Many authors have emphasized the presence of chaotic dynamics in a wide variety of communities with interacting species [34], such as outbreaks of planktons in the phytoplanktonzooplankton system, which is one of the important predatorprey systems. With the complex dynamics found in this research, many complicated states or oscillations of predatorprey populations in the real world may be possibly explained. Also, this research could be useful for researchers who will further explore the discrete-time predator-prey systems.

\section{Conflicts of Interest}

The authors declare that they have no conflicts of interest.

\section{Acknowledgments}

The authors would like to thank the National Major Science and Technology Program for Water Pollution Control and Treatment (no. 2017ZX07101-002) and the Fundamental Research Funds for the Central Universities (no. JB2017069) for their support.

\section{References}

[1] R. P. Gupta and P. Chandra, "Bifurcation analysis of modified Leslie-Gower predator-prey model with Michaelis-Menten type prey harvesting," Journal of Mathematical Analysis and Applications, vol. 398, no. 1, pp. 278-295, 2013.

[2] Z. M. He and X. Lai, "Bifurcation and chaotic behavior of a discrete-time predator-prey system," Nonlinear Analysis: Real World Applications, vol. 12, no. 1, pp. 403-417, 2011.

[3] Z. Ma, F. Chen, C. Wu, and W. Chen, "Dynamic behaviors of a Lotka-Volterra predator-prey model incorporating a prey refuge and predator mutual interference," Applied Mathematics and Computation, vol. 219, no. 15, pp. 7945-7953, 2013.

[4] P. J. Pal and P. K. Mandal, "Bifurcation analysis of a modified Leslie-Gower predator-prey model with Beddington-DeAngelis functional response and strong Allee effect," Mathematics and Computers in Simulation, vol. 97, pp. 123-146, 2014.

[5] H. N. Agiza, E. M. ELabbasy, H. EL-Metwally, and A. A. Elsadany, "Chaotic dynamics of a discrete prey-predator model with Holling type II," Nonlinear Analysis: Real World Applications, vol. 10, no. 1, pp. 116-129, 2009.

[6] Z. Y. Hu, Z. D. Teng, and L. Zhang, "Stability and bifurcation analysis of a discrete predator-prey model with nonmonotonic functional response," Nonlinear Analysis: Real World Applications, vol. 12, no. 4, pp. 2356-2377, 2011.

[7] A. J. Lotka, "Analytical note on certain rhythmic relations in organic systems," Proceedings of the National Acadamy of Sciences of the United States of America, vol. 6, no. 7, pp. 410415, 1920.

[8] V. Volterra, "Fluctuations in the abundance of a species considered mathematically," Nature, vol. 118, no. 2972, pp. 558-560, 1926.

[9] L. Stone and D. He, "Chaotic oscillations and cycles in multitrophic ecological systems," Journal of Theoretical Biology, vol. 248, no. 2, pp. 382-390, 2007.

[10] Y. Li and Y. Ye, "Multiple positive almost periodic solutions to an impulsive non-autonomous Lotka-Volterra predator-prey system with harvesting terms," Communications in Nonlinear Science and Numerical Simulation, vol. 18, no. 11, pp. 3190-3201, 2013.

[11] J. Sugie, R. Kohno, and R. Miyazaki, "On a predator-prey system of Holling type," Proceedings of the American Mathematical Society, vol. 125, no. 7, pp. 2041-2050, 1997.

[12] G. T. Skalski and J. F. Gilliam, "Functional responses with predator interference: viable alternatives to the Holling type II model," Ecology, vol. 82, no. 11, pp. 3083-3092, 2001.

[13] Z. Xiang, D. Long, and X. Song, "A delayed Lotka-Volterra model with birth pulse and impulsive effect at different moment on the prey," Applied Mathematics and Computation, vol. 219, no. 20, pp. 10263-10270, 2013.

[14] J. Vandermeer, "Seasonal isochronic forcing of Lotka-Volterra equations," Progress of Theoretical and Experimental Physics, vol. 96, no. 1, pp. 13-28, 1996. 
[15] S. Gakkhar and R. K. Naji, "Chaos in seasonally perturbed ratiodependent prey-predator system," Chaos, Solitons and Fractals, vol. 15, no. 1, pp. 107-118, 2003.

[16] H. Zhang, T. Huang, and L. Dai, "Nonlinear dynamic analysis and characteristics diagnosis of seasonally perturbed predator-prey systems," Communications in Nonlinear Science and Numerical Simulation, vol. 22, no. 1-3, pp. 407-419, 2015.

[17] D. C. Mistro, L. A. D. Rodrigues, and S. Petrovskii, "Spatiotemporal complexity of biological invasion in a space- and time-discrete predator-prey system with the strong Allee effect," Ecological Complexity, vol. 9, pp. 16-32, 2012.

[18] P. Comer, D. Faber-Langendoen, R. Evans et al., Ecological Systems of the United States: A Working Classification of U.S. Terrestrial Systems, vol. 223, Nature Serve, Arlington, Va, USA, 2003.

[19] X. L. Liu and D. M. Xiao, "Complex dynamic behaviors of a discrete-time predator-prey system," Chaos, Solitons and Fractals, vol. 32, no. 1, pp. 80-94, 2007.

[20] Z. J. Jing and J. P. Yang, "Bifurcation and chaos in discrete-time predator-prey system," Chaos, Solitons and Fractals, vol. 27, no. 1, pp. 259-277, 2006.

[21] T. Huang and H. Zhang, "Bifurcation, chaos and pattern formation in a space- and time-discrete predator-prey system," Chaos, Solitons and Fractals, vol. 91, pp. 92-107, 2016.

[22] P. P. Saratchandran, K. C. Ajithprasad, and K. P. Harikrishnan, "Numerical exploration of the parameter plane in a discrete predator-prey model," Ecological Complexity, vol. 21, pp.112-119, 2015.

[23] G. Domokos and I. Scheuring, "Discrete and continuous state population models in a noisy world," Journal of Theoretical Biology, vol. 227, no. 4, pp. 535-545, 2004.

[24] R. Yang, "Bifurcation analysis of a diffusive predator-prey system with Crowley-Martin functional response and delay," Chaos, Solitons and Fractals, vol. 95, pp. 131-139, 2017.

[25] P. H. Crowley and E. K. Martin, "Functional responses and interference within and between year classes of a dragonfly population," Journal of the North American Benthological Society, vol. 8, pp. 211-221, 1989.

[26] X.-Q. Liu, S.-M. Zhong, B.-D. Tian, and F.-X. Zheng, "Asymptotic properties of a stochastic predator-prey model with Crowley-Martin functional response," Applied Mathematics and Computation, vol. 43, no. 1-2, pp. 479-490, 2013.

[27] X.-Y. Meng, H.-F. Huo, H. Xiang, and Q.-Y. Yin, "Stability in a predator-prey model with Crowley-Martin function and stage structure for prey," Applied Mathematics and Computation, vol. 232, pp. 810-819, 2014.

[28] M. Sivakumar, K. Balachandran, and K. Karuppiah, "Bifurcation and spatiotemporal patterns of a density-dependent predator-prey model with Crowley-Martin functional response," International Journal of Biomathematics, vol. 10, no. 6, Article ID 1750079, 2017.

[29] Y. Y. Dong, S. L. Zhang, S. B. Li, and Y. L. Li, "Qualitative Analysis of a Predator-Prey Model with Crowley-Martin Functional Response," International Journal of Bifurcation and Chaos, vol. 25, no. 9, Article ID 1550110, 2015.

[30] S. B. Li, J. H. Wu, and Y. Y. Dong, "Uniqueness and stability of a predator-prey model with C-M functional response," Computers and Mathematics with Applications, vol. 69, no. 10, pp. 1080-1095, 2015.

[31] S. B. Li and J. H. Wu, "Qualitative analysis of a predatorprey model with predator saturation and competition," Acta Applicandae Mathematicae, vol. 141, pp. 165-185, 2016.
[32] J. Ren, L. Yu, and S. Siegmund, "Bifurcations and chaos in a discrete predator-prey model with Crowley-Martin functional response," Nonlinear Dynamics, vol. 90, no. 1, pp. 19-41, 2017.

[33] A. J. Nicholson, "The self-adjustment of populations to change," Cold Spring Harbor Symposia on Quantitative Biology, vol. 22, no. 0, pp. 153-173, 1957.

[34] A. Hastings, C. L. Hom, S. Ellner, P. Turchin, and H. C. J. Godfray, "Chaos in ecology: Is mother nature a strange attractor?" Annual Review of Ecology, Evolution, and Systematics, vol. 24, pp. 1-33, 1993. 


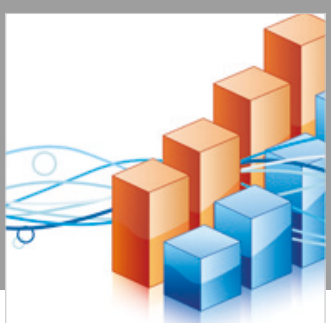

Advances in

Operations Research

\section{-n-m}
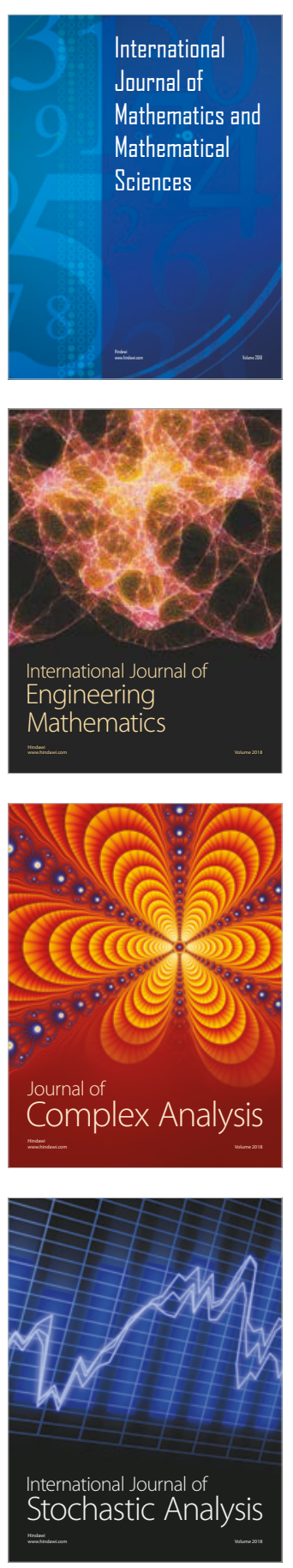
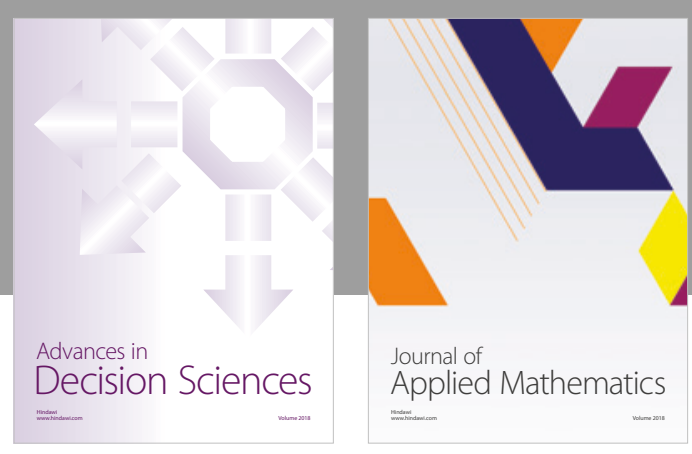

Journal of

Applied Mathematics
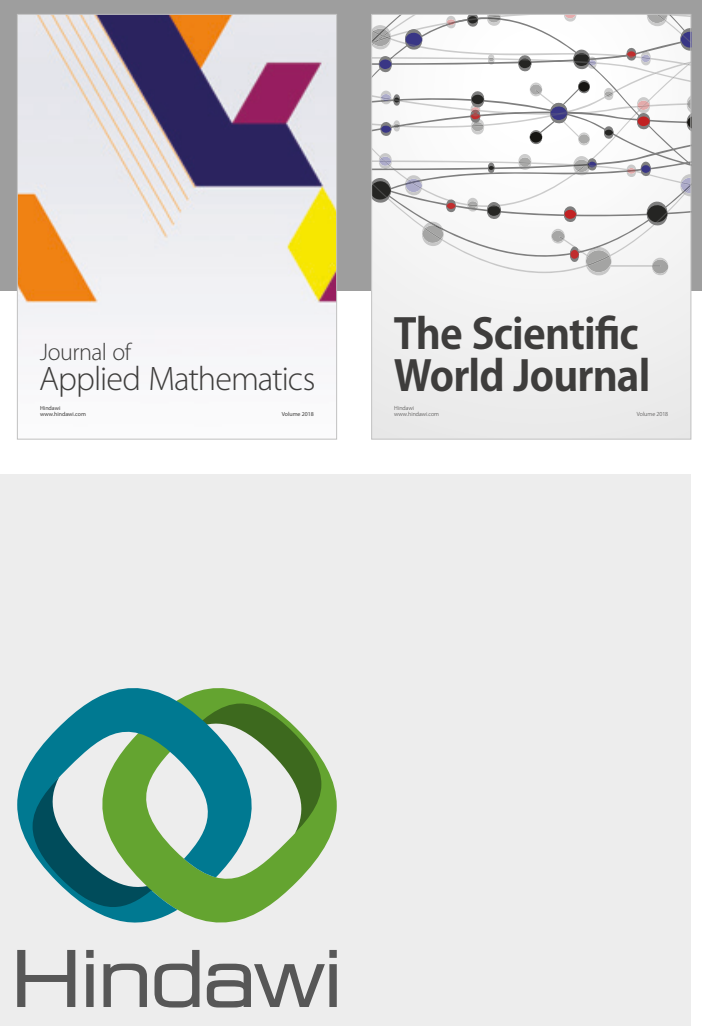

Submit your manuscripts at

www.hindawi.com

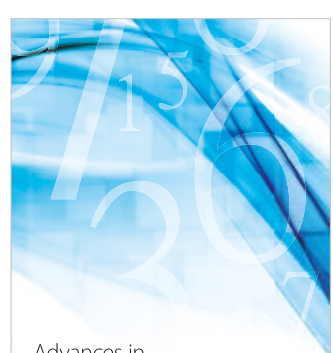

Advances in
Numerical Analysis
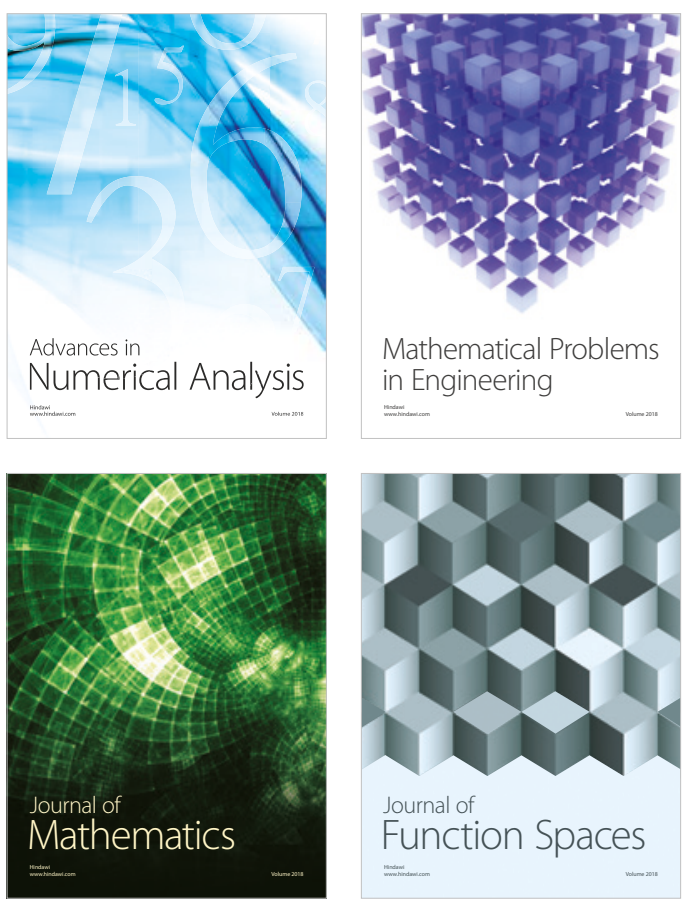

Mathematical Problems in Engineering

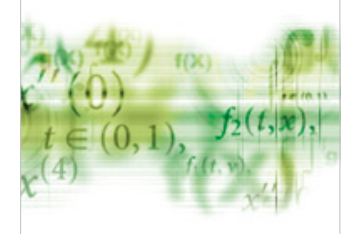

International Journal of

Differential Equations

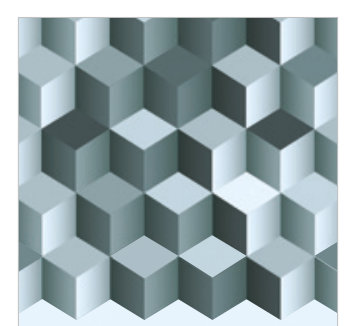

Journal of

Function Spaces
The Scientific

World Journal

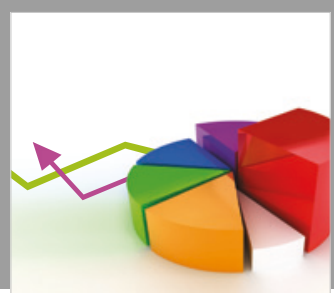

Journal of

Probability and Statistics
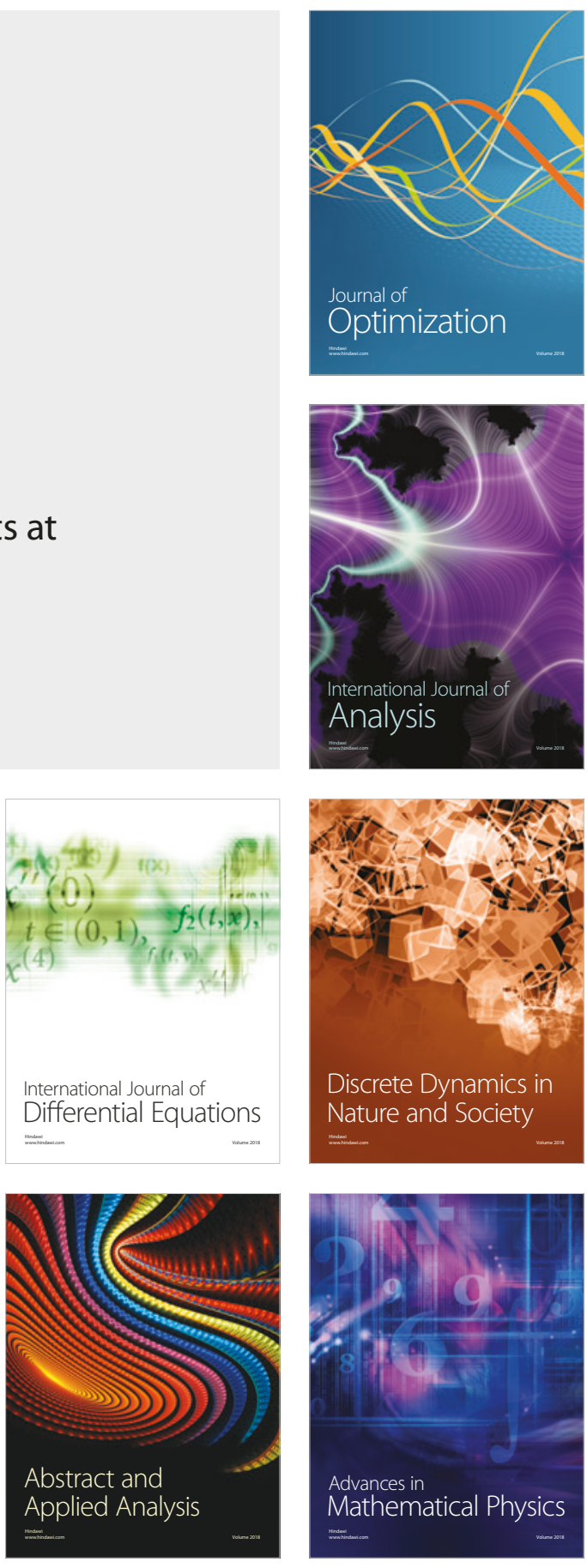